\title{
XV Annual Meeting of the International Society for Pediatric Neurosurgery, New York, 12-16 July 1987
}

\author{
Probability of intracranial complications in pediatric head \\ trauma: a multivariate analysis of risk factors. $\mathrm{K} . \mathrm{H}$. Chan, \\ C. P. Yue, and K. S. Mann (Hong Kong)
}

Among 12,072 hospital admitted head injured children, 159 developed intracranial complications which included hematomas, contusions, and diffuse brain swelling. We selected four clinical features as risk factors which may predict intracranial complications: impaired consciousness, vomiting, early post-traumatic epilepsy (EPTE), and skull fracture. Multivariate analysis using the stepwise logistic regression model showed that impaired consciousness ( $p 0.0001)$, vomiting $(p=0.006)$, and skull fracture ( $p 0.0001$ ) are significant, whereas EPTE is non-significant $(p=0.35)$. Presence of impaired consciousness plus skull fracture carries a $70 \%$ risk of intracranial complication. The risk is $18 \%$ for impaired consciousness alone. Skull fracture by itself has a relatively low risk of one percent. However, it is highly age-dependent: having a skull fracture is 16 times more dangerous in the teenagers than in infants. Vomiting per se has very low risk of less than $0.1 \%$. However, it is often associated with impaired consciousness and or skull fracture. Absence of any of the above risk factors is not entirely risk free: $0.06 \%$. Such analysis should be useful in formulating guidelines for the initial management of pediatric head trauma.

Comparative investigations in the multiple trauma injury of 73 children: two groups in two different countries. E. Markakis and A. Ahyai (Göttingen)

We report 73 children up to the age of 16 years with multiple trauma due to road traffic accident (RTA). Fifty-four children have been admitted to the Riyadh Central Hospital, Riyadh, Saudi Arabia (one year period: 1986), the hospital serving a population of 1.2 million. Ninteen children with multiple trauma injury after RTA have been admitted to the University Hospital of Göttingen over the period of three years (1984-1986, serving a population of 450 thousand). There are special characteristics in the age distribution, in the lethality rate, injury severity score, and the type of injury between the two groups. The number of ve- hicles is high in both countries, but attitude of driving and restricting laws, (e.g., obligatory safety belts, no sitting allowance of children in the front seats of the car in Germany, etc.) influence the number, type, severity and the outcome of injury.

Pediatric head injury - where to treat? W. L. Robinson (Baltimore, Md.)

It has been accepted as an article of faith that pediatric head injuries are best treated at specialized referral centers. Elaborate referral patterns to include ground based transportation, fixed wing aircraft, and helicopters have been established throughout the country. In an attempt to evaluate the question of where best to treat pediatric head injuries, a review of all patients admitted to the University of Maryland Medical System and related hospitals from 1985-1987 was carried out. The 610 patients in the study allowed analysis of injury type, severity, GCS score, treatment, treatment site, other injuries and outcome. In the total group, 30 patients died, with a death rate of 4.9. One hundred and ten operative procedures were carried out, with 26 deaths during or following surgical intervention. Seventyfour percent of all patients that died were patients with multiple systems injury. Closed head injury with cerebral concussion represented $50 \%$ of the cases, followed by intracranial hemorrhage, $14.3 \%$; basilar skull fracture, $12.3 \%$; cerebral contusion, 9.3\%; linear skull fractures, $7.6 \%$; gunshot wounds of the head, $4.7 \%$, and depressed skull fractures, $2.7 \%$. The most common operative procedure was intracranial monitoring with 55 subarachnoid screws being placed. Average length of stay in this series was 5.2 days with the LOS being negatively influenced because of the number of multiply injured patients seen in this study. Our data indicate that the vast majority of head injured children can be adequately cared for at the local community hospital, providing there is neurosurgical coverage and appropriate pediatric intensive care. Patients with severe brain injuries with multiple trauma or with anticipated medical/surgical complications should be transferred as soon as possible to a specialized unit. The immediate avail- 
ability of adjunct staff, the availability of ICU monitoring to include ICP and ready access to diagnostic facilities should prompt such transfers.

Early surgical management of diastatic linear skull fractures. S. Mutleur; L. Kostakoglu, and E. Tuncbay (Izmir)

During the years 1975-1986, 14,850 pediatric head injuries were admitted to the Aegean University Department of Neurosurgery. 3,102 of the cases had skull fractures and 44 of them showed diastatic linear fractures which were managed surgically. The criteria for the surgical treatment has been defined by Ramamurti et al. and Thompson et al. in the 1970s. In our series we had 44 diastatic linear skull fractures with widths greater than $4 \mathrm{~mm}$. The age of the cases ranged from 0-7 years (mean 2.9). Twenty-seven of the patients were male and 17 were female. The most common etiologic factor was a fall from a height $(88.6 \%)$ and the most common location of the fractures was the parietal region (86.4\%). The width of the fractures ranged from 4 to $14 \mathrm{~mm}$ (mean $6.09 \mathrm{~mm}$ ). The collected data from the patients' records was analysed. Cross-correlations and direct relationships among age, etiology, clinical and surgical findings, the associated lesions, prognosis and follow-up are made. As well, the relations amongst fractures, dural and cortical lacerations, early and late epilepsy and the effectiveness of early surgical management are discussed.

Assessing the conscious level in infants and young children: a paediatric version of the glasgow coma scale. D. A. Simpson, P. L. Reilly, R. Sprod, and L. Thomas (North Adelaide)

In most parts of the world, the conscious level is assessed by the Glasgow Coma Scale, in terms of eye opening, best motor response and best verbal response. This scale was designed for adults, but is also applicable to older children. It is not applicable to young children, and still less to infants, since the scale lays down norms of response which are not normally achievable by the very young. In 1982, two of us (PLR and DAS) briefly described a paediatric coma scale, which is basically very similar to the adult scale, with the important difference that the scale lays down age-related norms for best motor and best verbal responses which are lower than those of the normal adult. These norms are based on studies of the neurological development of normal infants and children. They are set out in the directions printed on the back of each paediatric coma scale sheet.

Posterior fossa epidural hematomas in children: the impact of neurosurgery and CT scanner on their management. K.S. Mann (Hong Kong)

Post-traumatic epidural hematomas of the posterior fossa are rare, when compared with the supratentorial hematomas in children, and there have been only occasional case reports in the literature. A review of head injury case records at our hospital revealed 10 cases with epidural hematomas in children below the age of 16 years. Three patients, ages 7, 10, and 14 years respectively, were admitted before neurosurgery or CT scanning services were established at our hospital. All three died, 15, 12, and 10 hours after admission to hospital. Despite the fact that supratentorial burr-holes were made, the diagnosis of posterior fossa epidural hematoma was made only at postmortem. Since the introduction of neurosurgery and CT scanning, however, we have successfully diagnosed and treated 7 children with posterior fossa epidural hematomas. There were 4 male and 3 female children and the mode of injury was a traffic accident in 2 , injury at playground in 3 , and a fall in 2 . Neck stiffness, pain or retraction was observed in 5 children, increasing headache was observed in 4, and repeated vomiting was present in 3. Skull x-ray revealed a linear skull fracture in six children and skull fracture was observed at operation in all seven. CT scan confirmed the diagnosis of epidural hematoma in all. Size of hematoma varied from $80-120 \mathrm{ml}$. All children survived, and 6 are now completely normal. One patient still has ataxic gait but he is slowly improving from residual symptoms.

Post-traumatic seizures in children: what and when to treat. Y. S. Hahn, S. Fuchs, A. M. Flannery, M. J. Barthel, and D. G. McLone (Chicago, Ill.)

The ideal treatment of children with head trauma would include prevention of post-traumatic seizures. We retrospectively analyzed data on 937 children admitted to The Children's Memorial Hospital between 1981-1986, with head trauma. Ninety-two $(9.8 \%)$ experienced post-traumatic seizures. In $94.5 \%$ of patients $(87 / 92)$ seizures developed within the first 24 hours of injury. Three children had convulsions at 24 hours and at seven days, but only two children developed seizures after the first week. Factors found to influence the likelihood of seizures included severe head injury, diffuse cerebral edema, and acute subdural hematoma. Severe head injury was defined as Coma Scale (GSC) or Children's Coma Scale (CCS) less than eight, and minor head injury as GCS/CCS greater than 13 . Severely head injured children had seizures in $35 \%$ of cases. Only $5.1 \%$ with minor head injury had convulsions. This difference was statistically significant $(P 0.001)$. Seizures also occurred to a statistically significant frequency $(P 0.001)$ in those children with documented CT evidence of diffuse cerebral edema, and acute subdural hematoma. A less significant correlation $(P 0.1)$ was noted between seizures and open depressed skull fractures. We found no significant correlation between seizure occurrence and numerous other factors including age, sex, fracture location and type (other than open, depressed fractures as above), parenchymal injuries, fixed neurological deficits, and cranial surgery. These data may allow us prophylactically to treat those patients at greatest risk for seizures, while spar- 
ing those unlikely to seize from the possible side effects of anticonvulsants.

Outcome in children with severe head injuries. J. E. Rodriguez, J. M. Portillo, and C. P. Diaz (Madrid)

Eighty children with severe head trauma and with a Glasgow Coma Score (GCS) under 8 are presented. We have studied several factors with prognostic value. The 80 cases were classified according to seven head CT scan morphological patterns: (1) isolated extracerebral hematoma, (2) extracerebral hematoma with underlying hemispheric swelling, (3) single brain contusion, (4) multiple brain contusion, (5) diffuse brain swelling, and (6) diffuse axonal injury. The intracranial pressure (ICP) was monitored in all the children. Treatment included mechanical ventilation and dexamethasone. Mannitol and thiopental were used if increased ICP was noted. The intra- and extracerebral hematomas were evacuated and the brain contusions were surgically resected. The results were analyzed after comparing the age, type of lesion, GCS and ICP. Conclusions: (1) diffuse brain swelling was the most common CT scan finding and the one with best prognosis; (2) age is a bad prognostic factor in children under two years old or younger; (3) an ICP maintained over $30 \mathrm{mmHg}$ is a bad prognostic factor; (4) extracerebral hematoma plus underlying hemispheric swelling and diffuse axonal injury were the two scan findings with the worst prognosis; and (5) in our opinion, the GCS has no prognostic value by itself. It should therefore be evaluated along with the above mentioned factors.

Criteria for diagnostic and intensive care of severe head injured children and follow-up of their psychophysical development. A. Gostisa and M. Korsic

The authors present the clinical (Glasgow Coma Score Frankfurt) and neurophysiological (SEP, ABR, EEG) criteria of severe head injury on admission of children into the hospital to develop the criteria for intensive care. The authors also present the results of neurological, neurophysiological, and psychological follow-up of 52 children in post-traumatic coma within $48 \mathrm{~h}$ after admission in intensive care unit. The results show $44 \%$ mortality $(80 \%$ in the group under 2 years, $29 \%$ in the age group 2 to 6 years and $47 \%$ in school children). The results of psychosocial disturbances of different groups of injured children will be given depending on the age and duration of coma.

Outcome from severe head injury in children and in different classes of age. M. Colangelo, A. Buonaguro, and A. Ambrosio (Naples)

A consecutive series of 96 children admitted to the Santobono Children's Hospital of Naples between 1978 and 1985 is presented. The patients ranged in age from 1 month to 6 years and they were grouped in two classes. The first (class I) comprised children from 1-36 months whereas the second (class II) included older children from 3-6 years. The study included those patients who remained comatose
- defined as a Glasgow Coma Scale (GCS) score of 8 or less as far as a Children Coma Scale (CCS) score of 10 or less - for at least 12 hours. The outcome was determined at least 3 months after injury and included the following categories: good recovery, moderate disability, severe disability, vegetative state and death. The data confirm a significant relationship between age and outcome. According to our data, morbidity was higher in younger children: $51 \%$ of class I patients had a severe disability, while the mortality rate was higher among the older children. The best recovery was observed among the toddler and older children. In addition, according to this retrospective study there is striking evidence that the predictive value of the CCS is lower than the one of the GCS. The authors emphasize the parameters which lead to a correct prediction of the outcome in the different age groups.

\section{Atlas assimilation: implications (A. H. Menezes (Iowa City,} Io.)

Assimilation of the atlas is a frequently encountered, but poorly understood abnormality of the craniovertebral junction (CVJ). Progressive problems range from atlanto-axial instability (AAI) to severe basilar invagination (BI) and soft tissue mass compromising the medulla. Thirty-six symptomatic patients were investigated to provide insight into the pathological history and management. Assimilation comprised $12 \%(36 / 296)$ of CVJ abnormalities treated. Presentation was 5-20 years, 14; 20-40 years, 17; $40-60$ years, 5. Symptoms included nuchal rigidity, brainstem- cervical myelopathy and cranial nerve dysfunction. Sleep apnea (5) and acute trauma precipitated deficits in 7. Basilar migraine and scoliosis were peculiar to children. Patients were evaluated by dynamic myelotomography, $\mathrm{CT}$, traction, MRI and at operation. The effective foramen magnum sagittal diameter was always less than $20 \mathrm{~mm}$. Segmentation failure $\mathrm{C} 2 / \mathrm{C} 3(30 / 36)$ and 28 had irreducible basilar invagination surrounded by granulation, necessitating ventral medullary decompression (24 had secondary dorsal fixation). Reducible AAI or BI was only in children below 14 years $(8 / 36)$, where no granulation was seen. It was irreducible in 6 older teenagers. Associated Arnold-Chiari Malformation (ACM) in 12/36 and hydromyelia, 3. Ten of $12 \mathrm{ACM}$ had immediate improvement with ventral decompression of BI. All patients had posterior atlantoaxial arthrodesis. Improvement was the rule. Conclusions: Assimilation with non-segmentation $\mathrm{C} 2 / \mathrm{C} 3$ (43\% ACM) leads to AAI in childhood. Subsequent odontoid upward invagination, and granulation proliferation from instability, leads to irreducible neural compromise. To arrest the disease process, attention must be focused on the pediatric age group.

Internal fixation for atlanto-axial instability in children. G. A. Flint and A. D. H. Hockley (Birmingham)

Cervical instability in childhood may result from several pathological processes, including infection, trauma, neo- 
plasm, and congenital anomaly. Traditional surgical treatment relies on bone grafting techniques to achieve fusion. In the past such methods have involved the use of external fixation apparatus post-operatively. The authors describe a new and simple technique which they have used successfully in adults for atlanto-axial subluxation due to rheumatoid disease. The technique involves a metal rectangle wired in place to act as an internal fixation device, with onlay bone grafts. This avoids the need for complex or prolonged external fixation methods.

Spondylarthritis in children. M. Choux, G. Bollini, J. L. Jouve, M. Panuel, G. Lena, and L. Genitori (Marseille)

We studied 21 children who presented symptomatic diskspace infection and vertebral osteomyelitis, with an average follow-up of 3 years and 6 months. At the same time, we analyzed 360 cases of diskitis in children from 27 series in the literature. Before the age of 4 years (13 cases) the symptoms are numerous, including septicemia (3 cases). After the age of 4 years ( 8 cases) the patients complained of back pain again. In 7 cases an MRI examination was done. Ten needle disk-space aspirations were performed, with 7 positive cultures. In most of the cases with a disk-space infection, MRI showed an abnormal aspect of the adjacent vertebral bodies. The term "spondylarthritis" seems more appropriate than "diskitis" or "vertebral osteomyelitis".

Management of hydromyelia. J. H. Wisoff and F. J. Epstein (New York, N.Y.)

The authors review their experience in the management of 17 children with hydromyelia over an 18 month period. The children had a Chiari I malformation and a secondary hydromyelia, 3 children had meningomyelocele and 4 children had distal syrinxes with tethered cords from occult dysraphism. All patients were investigated with preoperative MRI and intraoperative ultrasound. These neurodiagnostic examinations dictated the choice of surgical intervention. All patients with Chiari I had decompression of the hindbrain malformations, myelotomy with drainage of the cyst and placement of a stent. When the syrinx extended to the obex, as demonstrated by intraoperative ultrasound, the obex was plugged. The children with meningomyelocele had cyst-pleural shunts and the patients with distal syrinxes underwent terminal ventriculostomy. The classical presentation of dissociated sensory loss was present in only 3 patients. Scoliosis without neurological deficit, headache and Lhermitte's sign were common presentations. The patients with tethered cords were generally asymptomatic from their syrinx.

Spinal dysraphic syndrome associated with anorectal anomalies. A. M. Flannery, F. M. Karrer, J. G. Raffensperger, and D. G. McLone (Chicago, Ill.)

The early recognition and treatment of correctable lesions of the terminal spinal cord in patients with anorectal mal- formation may preserve important neurological functions. Twelve patients, since 1981, were found to have spinal dysraphic states associated with anorectal anomalies. Six children had cloacal extrophy and six had imperforate anus (2 with high rectovaginal fistula, 3 low, 1 unknown). The children with cloacal extrophy were all noted to have skin covered lumbosacral masses. Two of six children were born with neurological deficits and foot deformities. For two children, lower extremities neurological function deteriorated within 3 to 6 months of birth. The two children seen most recently were normal at birth, underwent surgery in infancy and have done well. All children had tethered cords with two myelocystoceles, three lipomeningomyeloceles and one lipoma. With imperforate anus, two children presented with lumbar dimples, four with vertebral anomalies which caused scoliosis in two cases. None of these children were born with neurological deficits. Two children presented with progressive motor weakness and improved after surgery. Two children underwent early surgery, before they developed deficits, and have done well. One child was untethered prior to fusion for scoliosis and the remaining youngster was evaluated but not operated upon. Surgical outcome was good. No child became worse. Of the 11 operated cases, 8 of 11 stabilized or remained normal and 3 of 11 improved. We advocate that children with anorectal and urogenital anomalies who are noted to have back masses, dimples, hemangiomas or vertebral anomalies be screened for evidence of lipomas, tethered cord, lipomeningomyelocele and myelocystocele.

A new hypothesis for myeloschisis: overgrowth and reopening - an experimental study. O. Shizuo, H. Saya, and S. Matsumoto (Kobe)

A new hypothesis for embryopathogenesis of myeloschisis is described on the basis of experimental studies analyzing the stage specificity and immunohistochemical/histological characteristics of the exposed neural tissue (placode). Myeloschisis developed in six fetuses among 205 chick embryos treated in various stages with teratogens including ethylnitrosourea (ENU), anticonvulsants and antipyretics. All but one case of associated cephalothoracopagus (conjointed twins, janus) demonstrated myeloschisis in the thoracic region with a lamina defect of 2 or 3 levels, and none was exposed to a teratogen prior to or within Hamilton and Hamberger $(\mathrm{H} \& \mathrm{H})$ stage 12 (postincubation $45-49 \mathrm{~h}$ ), when the neuropore closes. Immunohistochemical studies on the chick myeloschisis clearly indicated that neuronspecific enolase (NSE) - positive elements were extremely active only in the overgrown placode corresponding to the histological findings with Kluver-Barrera's (K \& B) special stain. These findings were compared with those for a human newborn case of myeloschisis. Our observations imply the possibility of another mechanism for the embryopathogenesis of myeloschisis, and we have named this the "overgrowth and reopening" hypothesis. 
Occult spinal dysraphism. N. M. L. Ramos and G. S. Ajler (Buenos Aires)

We have made a review of the main works on spina bifida occulta which has convinced us of the absolute necessity that for its understanding, we require an accurate knowledge of its embryogenesis. This will allow us to understand the physiopathological phenomena causing the various syndromes. This study is based on a series of 45 cases of spina bifida occulta which we reviewed and treated between 1978 and 1986, at the Children's General Hospital in Buenos Aires. We used the classification of spinal dysraphism described by James and Lassman. On its basis, we analysed age, pathological and clinical pictures, the diagnostic studies performed and treatment. The conclusions were: (1) the presence of "tumour" and cutaneous stigmata or other lesions over the midline of the back must alert us to the possibility of spina bifida occulta, (2) the patient must be carefully studied with CT Metrizamide myelography and NMR, especially in those cases which must be operated upon, e.g., lipoma, (3) the beginning of sensibility or vesico-urinary disorders of unknown cause compel us to discard spina bifida occulta, and (4) the right surgical treatment will prevent, correct, and improve the prognosis of these lesions.

Neurosurgical treatment of spinal lipomas in childhood. K. Sakamoto, N, Kobayashi, and N. Morota (Kobe)

In order to improve the prognosis of patients with spinal lipomas, removal of mass to decompress, sectioning of a tethered cord, and structural reconstruction in early stage of life have been proposed by some neurosurgeons. However these proposals are still controversial and opposed by others. During a period from May 1970 to March 1987, 89 patients with spinal lipoma were treated at Kobe Children's Hospital. Of these, 65 were under the age of one year. Thirty-seven were within first two months of life. These age distributions were the result of our policy in early surgical treatment to the lipomatous invasion to the conus and the cord associated with spina bifida occulta. This paper analyses the results and discusses operative techniques and strategies. Reasons for selection of the early surgery are: (1) much easier operation, i.e., less bleeding, shorter operation time, easier reconstructive laminotomy to prevent future scoliosis, easier removal of softer lipomatous mass, easier reconstruction of the conus and the cord after removal of mass; (2) no asymptomatic cases in children more than two years were encountered; (3) better postoperative improvement in nervous and urological disorders in early operation group than late operation group; (4) less improvement in urological disorders in late operation group. After surgery, 8 patients needed reoperation because of no improvement or recurrence of symptoms. Postoperative follow-up study must be carried out by the aid of periodic cystometry or other urological diagnostic methods because of its sensitivity.
Tethered cord syndrome of delayed onset following repair of meningomyelocele. N. Tamaki, K. Shirataki, Y. Shouse, and S. Matsumoto (Kobe)

From 1971 to 1986,58 cases of meningomyelocele in which a repair was undertaken shortly after birth were encountered. Out of 58 patients, 8 (13.8\%) presented progressive neurological deficits of delayed onset following repair of a meningomyelocele shortly after birth. The patients included 5 boys and 3 girls. The average age of the patients at diagnosis of tethered cord syndrome was 10.1 months. All the patients had undergone meningomyelocele repair shortly after birth. Onset of the syndrome was at the average age of 9.1 months, and the duration of the neurological worsening was 15.2 months. There was an increase in neurological deficits; progressive weakness of the lower extremities in 3 patients, deformities of the feet in 3, and lumbar and leg pain in 3. Metrizamide myelography was performed in 7 cases, and Metrizamide CT scanning in 6 . Magnetic resonance imaging (MRI) was done in 5 cases using a $0.15 \mathrm{~T}$ MR scanner. All patients had a low-lying cord attached posteriorly at the site of the repair. The cord lies posteriorly throughout its course. Hydrocephalus was associated in 7 cases, Chiari type II malformation in 6 . Surgical untethering was performed in 7 cases; 5 showed improvement and 2 were unchanged. Pathogenetic factors are also discussed comparing MR and clinical findings of 12 patients with MMC unassociated with tethered cord syndrome.

Occult spinal dysraphism in chinese children. S.-T. Chan and D. Fong (Hong Kong)

A retrospective review was carried out on 33 Chinese children with occult spinal dysraphism managed over the period 1971-1986. Patients above 14 years of age were excluded. There were 12 boys and 21 girls. The age at presentation ranged from 3 days to 14 years. The commonest presenting symptom (58\%) was the presence of cutaneous manifestations over the lumbosacral region. Twenty-seven percent presented with neuromusculoskeletal syndrome and 15\% with urinary and bowel problems. Cutaneous abnormality was present in all except 1 case. The commonest lesion being a soft subcutaneous lipoma (in 73\% cases). 10 cases had a history of recent deterioration of neurological function. Operation was performed in 25 cases and consisted of excision of lipoma and untethering of the cord. Techniques employed included intra-operative EMG and SEP monitoring, use of nerve stimulator and operating microscope to identify the nerve roots, and meticulous watertight closure of the dura. Postop complications included CSF leakage in 5 cases, superficial wound infection in 5 cases, and 1 case of meningitis and post-meningitic hydrocephalus. There has been no mortality. Immediately postoperative the neurological state was improved in 1 case, remained unchanged in 23 and worsened in 1 . Long term follow-up results were also analysed. We conclude that surgical treatment is safe in experienced hands and should be performed as soon as the diagnosis is made. 
BAEP in Chiari type II malformation. K. Mori, Y. Uchida, and T. Nishimura (Nankoku City)

Brainstem auditory evoked potentials (BAEP) were examined in follow-up of patients with meningomyelocele associated with hydrocephalus in order to obtain baseline information. A total of 17 patients was examined (of whom 6 were males and 11 females) with ages ranging from one day to 33 years (average $8.5 \mathrm{y}$ ). Shunting operation was performed in 13 patients. One of these developed disturbance of consciousness, lower cranial nerve involvement and respiratory difficulty. BAEPs were recorded using NEC-Sanei Signal Processor 7T-17. Potentials evoked by $85 \mathrm{~dB}$ click sound were summed 2,048 times and recorded. This was carried out more than two times in order to confirm reproductivity. Except for one case, there was no prolongation of latency of I wave. All cases had prolongation of II to V waves. In cases of hydrocephalus not shunted, the latency was markedly prolonged and deformity of the waves with lowering of their amplitude was more prominent. Analysis of the BAEP in the patient with Chiari type II malformation shows that brainstem dysfunction may be present functionally, corresponding to the morphological changes on CT scans.

Subtorcular encephaloceles. P. H. Chapman and V. Caviness (Boston, Mass.)

The author's experience with two operated infants with occipital encephalocele studied by MRI scan has prompted a critical review of the anatomic and pathologic information available on this entity. Based on this analysis, we conclude that the incidence of associated hindbrain anomalies is substantial and should be anticipated in the operative treatment of the usual case. When this occurs, certain anatomic features are regular and predictable: (1) the fluidfilled sac represents a diverticulum of the IV ventricle; (2) there is associated cerebellar or tectal dysplasia; (3) the extracranial hernia arises caudal to the torcula regardless of the size of the posterior fossa or the presence of occipital lobe within the sac. Other features of subtorcular encephaloceles are variable and bear no constant relationship to the essential anomaly: the posterior fossa may be large or small; the degree of brainstem and cerebellar distortion and herniation is inconstant; and supratentorial brain structures are variably included in the sac. One cannot anticipate the structural details of the anomaly on clinical grounds alone but must rely on radiographic studies, in particular MRI scanning, as illustrated by the present cases.

Childhood hydrocephalus associated with tectal lesions. T. Maugans, T. S. Park, W. S. Cail, and S. Newman (Charlottesville, Va.)

Four cases or childhood hydrocephalus associated with tectal enlargement are presented. Headache was the most common symptom. A paucity of neurological findings was observed with only one case presenting with the dorsal midbrain syndrome. Computed tomography suggested aqueductal stenosis but only magnetic resonance imaging reliably demonstrated the location and extent of the lesions. Each case demonstrated enlargement of the tectal plate with one having a large exophytic component. Previous studies suggest that these lesions may represent lowgrade gliomas, gliosis or hamartomas. All patients underwent CSF diversion and are doing well. In the case with an exophytic tumour, however, two unnecessary surgical procedures were performed before the availability of MRI as CT suggested that the tumor was arising from the cerebellar vermis or the pineal body. Serial MRI studies are planned for follow-up of each case. These cases suggest that childhood hydrocephalus associated with aqueductal stenosis should be evaluated with magnetic resonance imaging. As the lesions may represent static pathology or slow-growing tumors, serial examinations using this modality may represent the best means of following these patients, obviating the need to perform potentially hazardous diagnostic or therapeutic surgical procedures.

Slit-ventricle syndrome: review of $\mathbf{1 5}$ cases. A. Olivi and R. L. McLaurin (Cincinnati, Ohio)

The slit-ventricle syndrome (SVS), its presentation, pathophysiology, and treatment has been the subject of diverse opinions and recommendations during the past two decades. In an effort to define the clinical features of SVS and to make recommendations concerning management we have reviewed 15 cases treated by a fairly uniform technique during the past 5 years. The syndrome consists of: (1) intermittent, but self-limiting, episodes resembling shunt malfunction, usually lasting a few days; (2) non-filling of the pumping device after compression; and (3) a slit-like ventricular system on CT scan. In all but 2 patients the initial shunt was performed in infancy. The mean interval from the initial shunt to treatment of SVS was 6 years. The age range at onset of SVS varied from 2 to 17 years with a mean of 7 years. All patients in this series were relieved of symptoms by placement of an anti-siphon device and, in most patients, upgrading the valve resistance. Analysis of this series has led to these conclusions: (1) SVS is a characteristic clinical entity, usually distinguishable from persistent shunt malfunction and from low-pressure headache; (2) the pathogenesis is intermittent obstruction of the ventricular catheter; (3) there is no good evidence that changes of brain compliance or La Place principles apply; and (4) placement of anti-siphon device and upgrading valve resistance is effective treatment.

Ventriculomegaly in achondroplasia: significance and etiology. P. Steinbok, O. Flodmark, and J. G. Hall (Vancouver)

The significance and cause of ventriculomegaly in achondroplasia was investigated in four achondroplastic children. The intraventricular pressure was monitored over $24 \mathrm{~h}$ followed by intraventricular injection of radionuclide 
alone or in combination with water-soluble contrast. The intraventricular pressure was elevated and the reabsorption of CSF into the sagittal sinus was slow in all cases but there was no obstruction to CSF flow. The spinal subarachnoid space was well seen in all patients. Jugular venograms with pressure monitoring were performed in three patients (bilaterally in one). These studies confirmed a narrow jugular foramen in all patients with a significant pressure gradient $(3-10 \mathrm{mmHg})$ when pulling the catheter back from the sigmoid sinus through the foramen. A second gradient was found in the jugular vein in two patients at the level of the upper thoracic aperature. This gradient was 6 and $14 \mathrm{mmHg}$ respectively. Identical venograms and monitoring of the venous pressure in a control group showed no pressure gradients across the jugular foramen and smaller gradients $(2-5 \mathrm{mmHg})$ across the thoracic inlet. We conclude from these studies that ventriculomegaly in achondroplastic children represents hydrocephalus, which is likely secondary to raised intracranial venous pressure due to hemodynamically significant stenosis of the jugular foramen and, in some cases, the jugular vein in the thoracic aperature.

Microcephalic hydrocephalus: concept and pathophysiology. S. Matsumoto, S. Oi, N. Tamaki, and M. Kimura (Kobe)

The authors propose a possible new clinical entity of hydrocephalus, namely "microcephalic hydrocephalus". This is defined with the following tentative diagnostic criteria; (1) disturbed cerebrospinal fluid circulation; (2) obvious ventriculomegaly; (3) microcephaly with the head circumference constantly below $-2 \mathrm{SD}$; (4) increased intracranial pressure as present evidence or possible in the past; (5) psychomotor retardation, neurological deficits associated. In the retrospective review of 230 cases of hydrocephalus in children, nine cases met the above criteria. All patients were infants and the age at the time of diagnosis ranged from 2 days to 4 years. Underlying diseases were found in 3 patients and these included lissencephaly and congenital muscular dystrophy (Fukuyama type). There was one familial case occurring in female siblings. CSF dynamics study utilizing Metrizamide CT cisternography demonstrated possible aqueductal stenosis in 3 and communicating pattern in the rest. Intracranial pressure (ICP) monitoring revealed high base line pressure in all ranging from 7 to $33 \mathrm{mmHg}$ and remarkable pressure waves in some. Six patients were treated with shunt operation and most showed slit-like ventricles with remarkable cerebral mantle reconstitution postoperatively. This paper, which presents many unsolved problems in its pathophysiology, does not confirm but only presents a possible new clinical entity of hydrocephalus.

Occult hydrocephalus in children. C. Di Rocco, M. Caldarelli, A. Ceddia, A. Iannelli, and F. Velardi (Rome)

Thirty-two children with hydrocephalus clinically manifested late in life, and operated on at the Neurosurgi- cal Department of the Catholic University Medical School, Rome, in the period 1976-1986, are considered for the present study. This group represents $10 \%$ of all non-tumoral hydrocephalic patients surgically treated in the same period. Six children were between 2 and 4 years of age at the diagnosis, 13 between 5 and 10 years, and 13 between 11 and 15 years. Head circumference was abnormally increased in 19 subjects, but the increase was minimal $(+2$ percentiles) in 6 of them. Pyramidal signs, psychomotor retardation and gait disturbances were present in about one third of the cases. Ocular signs were found in $37.5 \%$ of the patients, though papilledema was found only in 4 subjects. Epilepsy occurred in 7 cases and vomiting and headache in 6 . Endocrinological dysfunction under the form of growth retardation (5 cases) and precocious puberty ( 1 case) was present in one fifth of the patients. In 12 cases the history suggested the possible cause of the ventricular dilation (perinatal distress, 5 cases; leptomeningitis, 3 cases; von Recklinghausen disease, 3 cases; untreated aneurysm of the vein of Galen, 1 case), while in the remaining 20 subjects no anamnestic relevant findings were reported. The neuroradiological examination revealed a triventricular dilatation in more than half of the cases; a tetraventricular dilation was demonstrated in 6 patients, and a bilateral ( 5 cases) or unilateral ( 3 cases) dilation in the remaining children. CSF shunting resulted in a complete recovery in 30 patients, while only an improvement was recorded in the remaining 2 children. Postoperative subdural hygromas were demonstrated in 5 cases, but only 3 required a surgical treatment because of recurrent neurological signs.

The significance of ventriculomegaly in the newborn with myelodysplasia. W. O. Bell, T. E. Sumner, F. M. Volberg (Winston-Salem, N.C.)

The incidence of hydrocephalus in the meningomyelocele population varies in reported series from 69 to $89 \%$. The spina bifida child rarely demonstrates signs of hydrocephalus at birth, and most commonly the meningomyelocele is closed and no ventricular shunting is done unless signs of hydrocephalus appear. However, previous observations by one of the authors have indicated that ventriculomegaly is commonly present at birth, even in neonates without overt hydrocephalus. Thus, palpating the anterior fontanelle and cranial sutures and measuring the head circumference may be misleading. With this in mind, we sought a means of identifying those neonates who would subsequently develop hydrocephalus. Thirty newborns with meningomyelocele who had cranial ultrasound examinations shortly after birth were reviewed. Dubowitz gestational age, birth weight, birth head circumference, head circumference percentile, and the lateral ventricular ratio (LVR) were analyzed for each patient in the series. The mean head circumference percentile was 48 ; the range was from less than the 10th percentile to greater than the 90th percentile. Despite these data, ventriculomegaly, as defined by an LVR of greater than 0.32 , was present on the initial ultrasound examination in all but two of the neo- 
nates. Excluding those two patients, ultrasound always demonstrated an elevated LVR. Pearson's correlation coefficient revealed that only one third of the elevated LVRs could be explained by the head circumference percentile. Later ventricular shunting was required for evolving hydrocephalus following meningomyelocele closure in all but three infants. We concluded that: (1) the clinical examination of the myelodysplastic neonate usually does not reveal evidence of hydrocephalus, and (2) ventriculomegaly, as defined by an LVR of greater than 0.32 on ultrasonography, is predictive for the later development of hydrocephalus following closure of the meningomyelocele.

Neurosurgical involvement in pediatric orthotopic liver transplantation. J. R. Mawk, B. W. Shaw, Jr., R. P. Wood, and L. Williams (Omaha, Neb.)

Between 19 July, 1985 and 15th March, 1987, 72 orthotopic liver transplantations were carried out at the University of Nebraska Medical Center. Thirty-five of these transplantations (49\%) were in patients less than 18 years old. No adult patient experienced any neurosurgical complication, although several underwent Ladd pressure monitoring pre-, intra- and post-operatively. Eight of 35 children experienced substantial neurological complications, and six of 35 required neurosurgical intervention. There were three intracerebral hemorrhages (1 hemorrhagic infarction, 1 lobar hematoma and one mixed subarachmoid-subduralintraparenchymal clot). Two of these children died, but one was a high quality survivor. One child developed a Candida brain abscess in a zone of probable septic infarction, but survived neurologically intact. One child developed a severe anoxic insult in the postoperative period, and ultimately required a shunt. Mantle collapse required drainage of subdural hygromas. This child is severely disabled. One child required pressure monitoring for postoperative cerebral edema due to failure of the transplanted liver. Although ICP was in good control, he suffered hypotension and became brain dead. The authors discuss the evolution of their own surveillance for neurosurgical complications in this population. Factors underlying the relatively high percentage of neurosurgical complications are reviewed. Children undergoing liver transplantation who develop neurosurgical complications should be managed very aggressively, because the neurological outcome may be excellent.

Epilepsy surgery in children: experience at the children's hospital of eastern Ontario, Ottawa, Canada. E. C. Ventureyra, D. Izukawa, D. Keene, P. Humphreys, and L. P. Ivan (Ottawa, Ont.)

From August, 1981 to September, 1986, surgical intervention was performed in a carefully selected group of 17 boys and 11 girls with medically intractable focal epilepsy (23 cases) or non-atrophic structural lesion and associated seizure focus ( 5 cases). Mean age at surgery was $14.2 \mathrm{y}$ with a mean interval of $6.5 \mathrm{y}$ between onset of epilepsy and operation. All patients were evaluated preoperatively by complete physical and neurological examination, interictal EEG's (nasopharyngeal/sphenoidal leads), ictal EEG's (seizure activation/telemetry/videotelemetry), CT scan, visual field testing, neuropsychological testing and WADA test (intracarotid sodium amytal). All surgical procedures were performed under general anaesthesia with electrocorticographic control, (ECOG), with depth electrodes and cortical mapping. Surgical procedures consisted of osteoplastic craniotomy with temporal lobectomy (15), excision of brain tumour and epileptogenic focus (5), frontal corticectomy (2), parietal corticectomy (3), frontal lobectomy (1), partial hemispherectomy (1), no resection (1). Postoperative results were obtained for a mean follow-up period of 23 months. 18 patients had complete abolition of seizures while another two had only occasional auras. Five patients showed a greater than $50 \%$ reduction in seizure frequency while only 3 obtained no benefit from surgery. There was no mortality or significant morbidity encountered in this series. Surgery is a safe, effective and underutilized therapeutic alternative in the management of childhood epilepsy.

Cerebral granulomatous lesions and epilepsy in children. J.-U. Choi, S.-S. Chung, and K.-C. Lee (Seoul)

The authors reviewed 72 patients with cerebral epileptogenic granulomatous lesions. Most cases presented with seizure. The most common location of lesions was parietal. On brain CT scans the lesions were nodular in 30\%, ringenhancing in 53\% and calcified in $17 \%$. The size of the lesions ranged from 0.5 to $2 \mathrm{~cm}$. Forty eight patients were followed by brain CT scan for periods between 6 months to 7 years. In 32 out of 48 patients the lesions disappeared spontaneously or decreased in size, but size of lesion did not change in 14 cases and increased in 2 cases. Stereotaxic biopsy was done in 4 patients; 3 showed evidence of chronic non-specific inflammation and one tuberculous granuloma.

Selective posterior rhizotomy for the treatment of spasticity: relationship of intraoperative EMG patterns to the postoperative course. R. Abbott, J. H. Wisoff, N. Spielholtz, and F. J. Epstein (New York, N.Y.)

Twenty patients with spastic cerebral palsy have been treated by selective posterior rhizotomy over the past six months. Intraoperative EMG was carried out with 5 channel monitoring to include both quadriceps and gastrocnemius. The fifth channel monitored either the right hamstring or anal sphincter activity. Additionally, right median nerve activity was monitored for any evoked activity resulting from our stimulating the cauda equina dorsal rootlets with a signal averager gated to the stimulator on the EMG machine. We observed several characteristic patterns of abnormal response when stimulating the component rootlets 
of the L2-S1 dorsal roots in these patients. These response can be subdivided into two components; that which is obtained during stimulation of the rootlet and that which persists afterwards. We observed, as have others, both tetanic contraction and spread of activity to involve antagonistic muscles and muscles outside the myotactic unit. We also observed other abnormal responses within the synergistic muscle groups; specifically, loss of fatiguing in the muscle during stimulus trains and an irregular electrical response in the musculature suggesting polysynaptic transmission. Surgical and monitoring technique will be discussed and the intraoperative EMG pattern types will be correlated with the post-operative course of the patients in an attempt to grade the degree of abnormality of these patterns thus increasing their intra-operative utility.

Injection injury of the sciatic nerve (370 cases). F. Villarejo, C. Morales, C. Gonzalez, C. Perla, and A. Isla (Madrid)

Injury to peripheral nerves complicating injections of therapeutic and other agents is common. The postulated mechanism of injury includes direct needle trauma, secondary constriction by scar, and direct nerve fiber damage by neurotoxic chemicals in the agent injected. Neurological sequelae can range from minor, transient, sensory disturbance to severe sensory disturbance and motor parlysis with poor recovery. The recommended treatment has ranged from a conservative approach of non-intervention to immediate operative exposure and irrigation, and has included early neurolysis and delayed exploration with neurolysis or resection. Recent experimental studies examining the effects of a wide variety of agents implicated in injection accidents have added to our understanding of the pathophysiology of this condition and have helped provide a basis for treatment. The sciatic and radial nerves are, by far, the most commonly affected. Clinical features include the immediate onset of severe pain at the site of injection with, often, radiation along the course of the nerve affected. Motor and sensory disturbance, which may be partial or complete, follows rapidly. The site of drug injection is the crucial factor in determining the degree of nerve fiber injury. Most moderate or severe injection injuries are the result of direct intrafascicular injection into the nerve trunk. The nature of the injected compound is also important, as certain drugs are much more damaging than others when injected into a peripheral nerve. We are presenting 370 cases of injection injury of the sciatic nerve in children treated during the last 20 years at the Neurosurgical Department of La Paz Hospital in Madrid. Pathology, clinical course, treatment and results are discussed.

Results in the management of craniopharyngioma in children: an endocrinological approach to treatment. J. $\mathrm{K}$. Kang, C. K. Park, M. C. Kim, and J. U. Song (Seoul)

Twenty-one patients with craniopharyngiomas were treated during a 10 -year period. Of the 21 patients, 15 were males and 6 were females, with an age range 3 to 45 years. The authors reviewed 14 patients less than 18 years of age, who presented with craniopharyngioma. In this study the mean follow-up period was 5 years. In children, the most common presentation was the endocrinological deficits $(85 \%)$, with the most frequent preoperative endocrine dysfunctions being growth failure $(35.7 \%)$, diabetes insipidus (21.4\%) and hypogonadism with obesity present in $28.6 \%$ preoperatively. Visual dysfunction symptoms were present in $70 \%$ of the patients. Patients were treated by: (1) subtotal excision; (2) subtotal excision followed by radiotherapy; (3) total excision; (4) total excision followed by radiotherapy. No recurrences have occurred in total excision with radiotherapy, and symptomatic recurrences occurred in $7 \%$ treated by subtotal removal followed by radiotherapy (mean time 6 months). The results of therapy indicated that total excision was deemed to have been achieved in only seven patients, six of whom have no recurrence. However, $78 \%$ of patients are in remission, $3 \mathrm{had}$ a good result (21\%), 8 had fair results (57\%). Two patients (28\%) died 3 years after total and subtotal excisions followed by radiation therapy. All of the children manifested hormonal deficiencies postoperatively and the majority had panhypopituitarism with diminished antidiuretic, adrenal, thyroid, growth and gonadotrophic hormones. Of the 14 patients, 6 were diabetes insipidus (42\%), 4 were growth hormone deficiency (28\%) and 4 were hypogonadism (28\%). Properly treated endocrine deficient children do well in terms of survival and endocrine deficits do not impair the quality of survival.

\section{Reoperation in the management of childhood cranio- pharyngioma. P. W. Carmel (New York, N.Y.)}

Even some advocates of initial radical removal of craniopharyngiomas have felt reoperation was "precluded because of prohibitive morbidity and mortality". In the period from 1977-1986, 46 children and adolescents were operated for craniopharyngioma at the Neurological Institute of New York. This was the initial procedure for 36 children, while 10 had been treated elsewhere. Seven of the initial procedure group were subsequently reoperated. Thus, seventeen patients undergoing secondary operations form the basis of this report. They had previously undergone a total of 22 operations, and eight had been given $\mathrm{x}$-ray therapy. (An additional patient, previously treated with proton-beam irradiation, will be shown.) All patients but one had radical tumor removal. There was no operative mortality. Two patients had decreased vision postoperatively, while vision was improved in eight. All patients had postop diabetes insipidus. One child is in a chronic care hospital. Total removal was felt to be obtained in nine cases; four children with subtotally removed tumors subsequently had $x$-ray therapy. Our results indicate that neither prior operation nor $x$-ray therapy is an insurmountable obstacle to successful reoperation. The limits of reoperation will be described. Children with initial sub- 
total removal of craniopharyngioma are potentially curable, and radiation may be avoided or deferred.

Brain tumors in neonates and infants: study of 76 operated cases. C. L. Lapras, J. N. Guilburd, J. Guyotat, and J. D. Patet (Lyon)

Seventy-six infants with brain tumors were operated on in our department. Histology was: 12 benign; 35 low grade; 29 high grade, malignant tumors. Eleven out of 12 benign tumors were totally removed, 1 subtotally, without surgical mortality. No oncological treatment was administered and no recurrences were observed. Eight cases are free of neurologic deficit, 4 present a mild or severe neurological sequela. No psychological impairment is observed. In the highly malignant tumor group, total excision was performed in 16 cases, subtotal in 6 , partial in 7 . Surgical mortality was $5 / 29$. The remaining 24 cases received oncological treatment but only 5 of them survived late. They were medulloblastomas: 4 underwent a total and 1 a subtotal excision of tumor. All received chemotherapy; 4 received radiotherapy also. Two children are free of sequelae, 3 others are retarded and have a short stature. In the group of low grade malignancy (35), 17 were totally removed, 7 subtotally, 5 partially, and 6 had a biopsy. The surgical mortality was $5 / 35$. Of 19 cases not receiving oncological treatment, 15 survived late ( 2 with a severe and 4 a mild deficit, 9 without sequelae). Three cases out of 11 receiving oncological treatment survived (2 without neurological deficit, 1 with a mild sequela). In infancy, the functional recovery of a focal surgical lesion, even extensive, is more effective than that of a diffuse lesion caused by radiotherapy. On this basis and according with the results of the present series, we emphasize the importance of radical surgery in the treatment of brain tumors in neonates and infants, with oncological treatment reserved for highly malignant or infiltrative tumors.

Benign astrocytomas and oligodendrogliomas of the hemispheres in childhood. J. F. Hirsch, A. Pierre-Kahn, E. Hoppe-Hirsch, C. Sainte Rose, and D. Renier (Paris)

Thirty-five benign astrocytomas and oligodendrogliomas of the cerebral hemispheres in children less than fifteen years of age were operated on between 1975 and 1986 (mean age 9 years, range $1-15$ ). There were 23 boys and 12 girls. The mean follow up in the series was $5.3 \mathrm{y}$. Seventy-seven percent of the cases presented with epileptic seizures. The delay between onset of symptoms and diagnosis varied between 15 days and 12 years (mean delay 3.3 years). Epilepsy was the only clinical feature in 22 patients $(63 \%)$. Tumors were located in the temporal lobe in $37 \%$ of the cases, in the frontal lobe in $31 \%$ and in the central region in $23 \%$. There were 20 astrocytomas (grade 1 or 2), 8 oligoastrocytomas, and 7 oligodendrogliomas. During the same period, 6 other benign tumors of the hemispheres were operated upon. Benign astrocytomas and oligodendro- gliomas constitute $85 \%$ of this total group. Tumor removal was considered as total in 33 children, subtotal in 1 , and partial in 1. Three patients died after surgery. Neurological sequelae were not common. Two children developed partial motor deficits and one developed a quadranopsia. The mean post-operative IQ was 90 . The two points of this study are: (1) surgery alone cured most of these tumors; although only 2 children were given radiotherapy, the recurrence rate was low (2 patients, i.e., 6\%); (2) epileptic seizures were cured in $86 \%$ of the children, although no specific surgical measures to treat epilepsy were made at the time of initial tumor removal. Sixty percent of the patients no longer receive antiepileptic medication.

Spectrum of non-pineal cell tumors arising in the pineal region. E. D. Friedman, P. W. Carmel, M. R. Fetell, and B. M. Stein (New York, N.Y.)

Pineal region tumors, although rare, are extremely diverse and remain controversial in their management. From a series of over 60 pineal region tumors, we have reviewed 23 non-pineal cell tumors in the pediatric population. All children have undergone tumor debulking and confirmation of histologic diagnosis. The spectrum of tumor types includes germinoma, teratoma, choriocarcinoma, embryonal cell carcinoma, endodermal sinus tumor, melanotic neuroectodermal tumor, epidermoid, and astrocytoma. Ages ranged from 3 months to 21 years, peak incidence 13-15 years, and a male predominance. CT was negative in two patients, but MRI diagnosed a leasion in each case. CT characteristics predictive of a poor outcome include: (1) intratumoral hemorrhage; (2) hyperdense tumor pre-contrast; (3) ependymal enhancement; and (4) rapid growth between scans. All germinomas were debulked, evaluated for CSF seeding and irradiated. Morbidity of surgery was minimal, but 4 patients died ( 2 postop). In three patients minute foci of malignant tumor was uncovered in serial sections of a germinoma, thereby directing a change in therapy. It is imperative that surgery be as complete a debulking as possible so aggressive malignancy may not escape detection. Chemotherapy to residual tumor may then prolong survival. If surgery confirms a benign growth, then the developing brain may be spared radiation and its longterm sequelae.

Retrospective analysis of 27 children with intracranial germ cell tumors (GCTs) experienced during the last 15 years. K. Sato, A. Ishizawa, S. Ishii, and H. Sumie (Tokyo)

Before the availability of CT, serum HCG and AFP measurement, and chemotherapy with the combination of cis-Platinum, Vinblastine and Bleomycin (PVB), highly radiosensitive tumors to 1,500 rads of "prove radiotherapy" and tumors poorly sensitive to the same were presumptively diagnosed retrospectively as germinoma and as malignant GCTs. At our institution, the former were treated by radiotherapy alone and the latter were treated by 
radical tumor resection followed by radiotherapy at that time. Diagnosis of GCTs experienced since then was usually confirmed by pathological features of tumors obtained during surgery as primary treatment, except for a case with $\mathrm{GCT}$ in the basal ganglion where neuroradiological and serum biochemical tests were the only tools for diagnosis. Postoperative radiotherapy alone for germinoma, postoperative chemotherapy with PVB for malignant GCTs, and chemotherapy alone for the surgically inaccessible GCT were chosen as adjuvant therapy. In our series, 6 histologically verified and 9 unverified germinomas, 8 histologically verified and 4 unverified malignant GCTs, and 1 possible malignant GCT in the basal ganglion, were included. The malignant GCTs included 2 malignant teratomas, 2 embryonal carcinomas, 3 choriocarcinomas, 1 endodermal sinus tumor, 3 mixed GCTs and 1 histologically unknown tumor with precocious puberty. Fourteen out of the 15 germinomas treated by radiotherapy with and without surgery survived during the observation period of between 8 and 176 months, with 1 exception where the patient died of tumor dissemination 29 months after the first admission. All 8 malignant GCTs treated by radiotherapy with and without surgery died, with a mean survival time of 20.4 months. Two out of 3 malignant GCTs treated by surgery following chemotherapy died with a mean survival time of 33.7 months, and the case with basal ganglion tumor treated by chemotherapy alone died of pneumonitis 35 months after the initiation of chemotherapy. By reviewing these cases, it was found that although thorough histological and histochemical examination of a tumor specimen may overlook a malignant component concealed in benign GCTs, at present all surgically accessible intracranial GCTs should be treated by radical tumor resection (in order to remove tumor mass as well as to establish histological features of the tumor) and followed by radiotherapy for germinoma and chemotherapy for malignant GCTs.

Chemotherapy of intracranial endodermal sinus tumors and recurrent germinoma with intracranial and spinal dissemination. T.-T. Wong, L.-S. Lee, Y.-L. Shieh, and L.-L. Law (Taipei)

For the treatment of recurrent intracranial germinoma and malignant germ cell tumors, we administer polydrug chemotherapy. We follow the "UCSF CCG-821" regimen (vinblastine, bleomycin, cisplatin, VP-16) for systemic chemotherapy, and we use additional intrathecal chemotherapy (methotrexate, Ara-C, hydrocortisone) for tumors with leptomeningeal disseminations. In the past one and a half years, we treated a 4-year-old boy who had a pineal germinoma with posterior fossa and spinal leptomeningeal disseminations by the use of this regimen with complete resolution of the recurrent tumors. We also treated 2 children with suprasellar endodermal sinus tumors by the use of systemic polydrug regimen alone. A 12-year-old boy with a recurrent mixed endodermal sinus tumor responded completely after 8 courses of chemotherapy. An 11-year- old girl with a pure endodermal sinus tumor showed partial response after 6 courses of systemic chemotherapy. All three of these children tolerated the treatment very well and remain alive and healthy.

Effective chemotherapy for malignant pineal germ cell neoplasms. C. Raffel,, R. Hudgins, V. Levin, A. Ablin, and M. S. B. Edwards (San Francisco, Calif.)

Fifteen patients with malignant pineal germ cell neoplasms treated by the Neurooncology Service at the University of California, San Francisco Medical Center from 1974-1987 have been reviewed. The average age was 12.6 years. Thirteen of the patients were males. The most common presenting symptoms and signs were headache, nausea, vomiting, papilledema and Parinaud's syndrome. Histologic confirmation of tumor type was obtained in thirteen patients. The other two patients were treated without biopsy because of increased CSF levels of AFP. Six patients had elevations of CSF AFP and/or B-HCG, five had no markers present in the CSF, and three patients had no CSF examination. Twelve patients were treated with radiation therapy and adjuvant chemotherapy; three patients received chemotherapy alone. Initially, patients were treated with chemotherapeutic regimens devised for primary CNS tumors. These four patients died with a mean survival of 3.25 years from diagnosis. More recently, patients have been treated with regimens devised for extra-CNS germ cell tumors. Of the 11 patients receiving germ cell protocols, an objective clinical and radiographic response was seen in all eight patients treated with a four-drug regimen of bleomycin, cis-platinum, vinblastine, and VP-16. Three of these patients received no radiation therapy. Of the eight patients, two suffered recurrences and died. Both required a marked reduction in the dose of the chemotherapeutic agents because of toxicity. The other six patients are alive and well. We believe that the regimen of bleomycin, cisplatinum, vinblastine, and VP-16 has promise as the primary treatment of malignant pineal germ cell tumors.

Treatment of malignant glioma with intratumoral instillation of autologus lymphocyte. S. N. Bhagwati (Bombay)

A 15-year-old girl was operated on in September, 1982, for a left fronto-parietal glioma and received 5,000 rads thereafter. Subsequently, she was given five courses of BCNU intravenously. She developed dysphasia and minimal weakness of the right upper limb 19 months postoperatively. CT scan showed recurrence of the tumor with the cyst around it in the left paraventricular region. An Ommaya reservoir was inserted and the catheter was left in the cystic cavity. A cell separator was used to recover autologous lymphocytes which were instilled into the cyst on three occasions over the next 13 months. She remained well for 26 months after the initial treatment, having had complete regression of her symptoms. However, she experienced headache, dysphasia, and right hemiparesis two and a quarter 
years after initiation of this treatment. CT scan showed recurrence of her tumor which had completely filled up the cystic cavity. She was then put on steroids with which she has maintained improvement. With intratumoral instillation of autologus lymphocytes, the patient has had complete regression of her symptoms for nearly two and a quarter years.

Personality and behavioural changes associated with tumors of the IV ventricle and brainstem. E. Brown, P. Slobogin, and F. J. Epstein (New York, N.Y.)

In this study we report on 25 pediatric patients with tumors of the IV ventricle or brainstem. In all cases the patients had multiple neurologic signs of brainstem dysfunction. Marked personality changes were noted in 21 of 26 cases, either pre- or postoperatively. Two distinct personality syndromes were observed and evaluated with neuropsychological assessment. Preoperatively, 7 of the 26 children presented initially with personality changes. These were highly variable but included irritability, emotional outbursts on provocation, manic responses, mood swings and deteriorating social relations. Postoperatively, 21 of 26 children showed marked personality changes. This was most dramatically seen in the midline cerebellar IV ventricle cases where the syndrome did not develop until 2-4 days following surgery. Presumably via focal edema, the patients developed multiple cranial nerve signs, including dysphagia and dysarthria. With this came marked behavioral changes including regressiveness, decreased verbalization and vocalization, primitive unmodulated emotional responses to any stimulation, excessive fearfulness, and a demanding style of social interaction. We interpret this as suggesting that reticular activating system and its limbic connections was at least partially disconnected from cortical control or modulation. In some instances the syndrome persisted for several months before subsiding somewhat.

An unusual mixed glioma with vascular malformations - an embryonal neoplasm? J. Cappelen, T. B. Halvorsen, L. G. Dale, and G. Nilsen (Trondheim)

A 6-year-old girl was referred with a four week history of diplopia and slight headache. On clinical examination she presented with anisocoria, strabismus, and papilledema. Radiological evaluation (CT, NMR, angiography) disclosed a huge cystic tumor in the right anterior fossa with midline shift. The tumor nodule was calcified and located in the bottom of this cyst. NMR and angiography demonstrated a network of great vessels with rapid shunting. At operation, the tumor presented as bright yellow, soft, partly necrotic mass with an unusual rich vascular supply of arteries, veins and aneurysmal dilatations. The cyst was located deep within the brain parenchyma and the tumor wall was demarcated from adjacent brain even though there was not exact cleavage plane. Medially, it was di- rectly continuous with the lateral ventricular wall. The histological picture has caused considerable difficulties in classification. The tumor was highly vascular. Pleomorphic cells with some atypia and few mitoses both of astrocytic and oligodendroglial origin was observed, as well as perivascular lipid macrophages and calcifications. On this basis we have classified the tumor as a mixed glioma and question whether this is a manifestation of progressive maturation in an embryonal neoplasm as proposed by Epstein et al.

Benign tumors of the endocranium extending into the brain stem and surgically treated in the late stages of clinical evolution. M. Mirko, V. Mile, R. Petar, B. Roza (Skopje)

In the course of the last two decades (1967-1986) we have operated on 159 children with endocranial tumors with supra- and infratentorial localization. Thirty-four children had benign tumors, especially with those that extend into the brainstem and its surrounding structures, which were compressed, adherent and infiltrated. The histological structure and evolution has especially influenced the surgical therapy. The clinical performance is most different and interesting. It depends on the localization and gross and histological features. In our series, the observed characteristics are late phase with disturbed consciousness, vital signs and manifest clinical symptoms of decreased function of the brainstem. We stated the indication for surgical treatment on the basis of diagnosis and concomitant hydrocephalus. Some patients were treated with ventriculo-peritoneal shunt and extirpation of the tumor afterwards. In 34 children (22\%), we had: grade I-II astrocytomas; craniopharyngiomas situated retro- or suprasellar; germinoma; IV ventricle papilloma; angioma; and, osteoma in the clivus. Twenty-nine children survived and 5 died $(15 \%$ mortality). The postoperative controls showed good results in terms of survival and functioning brain and brain stem.

Primitive fibromatous tumor in a 4-month-old white baby. Optic ultrastructural study. E. Cardia, M. Cambria, and S. Galatioto (Messina)

The present case refers to a 4-month-old patient in whom, by CT scan, an extensive lesion in the left fronto-temporoparietal region was discovered. This showed a great cystic part in which a solid mural nodule with a subdural attachment was detected. A complete surgical excision was possible. The cut surface of the tumor was whitish. Histological finding showed a fibromatous-like pattern in which parenchymal cerebral isles with normal light appearance were present. These latter data suggested the congenital origin of this rare tumor which during its growth could embed the normal areas of cerebral parenchyma. Our histological findings confirmed the congenital origin of such tumors as in the literature about similar cases. 
The Chiari II malformation and magnetic resonance imaging. R. M. Scott, S. M. Wolpert, M. L. Anderson, and E. B. K. Kwan (Boston, Mass.)

Cranial magnetic resonance imaging (MRI) was carried out in twenty-seven children with meningomyelocele to determine the potential of this imaging technique to display the pathologic anatomy of the Chiari II malformation, and to determine if correlation could be made between the anatomy displayed and the clinical signs and symptoms of the patients. MRI provided a striking depiction of the classic anatomic aberrations of this syndrome, but many of the abnormalities were not anticipated. For example, many patients had a capacious foramen magnum and wide CSF spaces anterior to the cord and brainstem at the foramen magnum. Partial agenesis of the corpus calosum was frequently seen; large syrinxes and other cystic collections of fluid within the IV ventricle or brainstem at the cervicalmedullary junction were clinically silent. One of the four patients symptomatic at the time of the scan had severe downward distortion of a malformed brainstem, unaccompanied by compression at the foramen magnum. Standard surgical decompression would not be anticipated to relieve this patient's symptoms. Thus, this imaging technique may play an important role in the evaluation of the symptomatic Chiari II patient in determining the place of surgical intervention.

Magnetic resonance imaging of mild and moderate head injuries in children. T. G. Luerssen, R. M. Ruff, J. R. Hesselink, C. A. Grote, L. F. Marshall, and M. R. Klauber (San Diego, Calif.)

The head injury research group at UCSD has been investigating factors which affect the ultimate outcome from pediatric head injury. One focus of these studies concerns the importance of magnetic resonance imaging (MRI) for detecting specific regions of brain injury and correlating these regions to neurologic and neurocognitive outcome. Work in the mild (GCS 13-15) and moderately (GCS 9-12) injured children has indicated that MRI can detect areas of brain injury where CT scans have not. These areas of injury are usually isolated and focal and can be either peripheral, involving cortical gray or peripheral white matter; or central, involving central white matter or deep gray nuclei. The neurologic and neurocognitive presentations are remarkably different for central lesions than for peripheral lesions. Furthermore, neurologic and neuropsychological improvement correlates temporally with resolution of the abnormalities seen on MRI. This pilot study demonstrates: (1) MRI can detect and localize abnormalities of brain parenchyma that correlate to neurologic deficit and may localize overt neurocognitive disturbance; (2) central white matter injuries are detectable by MRI in mild and moderately head injured patients; (3) central injury produces a clinical syndrome different from peripheral injury; (4) detection and localization of areas of brain injury with
MRI may help direct therapies aimed at remediation of head injured children.

MRI in the shaken baby syndrome. A.-C. Duhaime, R. A. Zimmerman, L. N. Sutton, and L. Schut (Philadelphia, Pa.)

Eight children under two years of age with the so-called "shaken baby syndrome" (unexplained trauma, subdural hemorrhages, retinal hemorrhages) were studied by acute and follow-up nuclear magnetic resonance (MRI) scans in an attempt to define better the pathophysiology and biomechanics of this injury in survivors. Acute studies showed blood in the subdural space, most often in the posterior occipital area and over the occipital convexity, which was more apparent than that seen on CT. In addition, an area of gyral enhancement was seen in the occipital cortex in five patients. Subacute studies showed changes consistent with a more diffuse hemorrhagic necrosis of the cortex. Follow-up studies disclosed progressive and severe atrophy of the cortex and white matter, particularly posteriorly, with the formation of large ex-vacuo extracerebral fluid collections. We believe these studies corroborate that a high acceleration impact-type injury with resultant cortical shearing is the cause of the severe brain damage seen in this syndrome.

Reversal sign: a pattern of diffuse hypoxic/ischemic cerebral injury. B. K. Han, R. B. Towbin, W. S. Ball, Jr., R. L. McLaurin, and W.-K. Jaun (Cincinnati, Ohio)

The reversal sign, a striking CT finding, probably represents a pattern of diffuse, hypoxic/ischemic cerebral injury. CT features of the reversal sign are diffusely decreased density of the cerebral cortical gray matter and white matter with a decreased or lost gray/white matter interface, or reversal of the gray/white matter densities and relatively increased density of the thalami, brainstem, and cerebellum. The etiology of this finding is not clear. It may be related to anatomic, vascular, chemical and metabolic factors. CT and medical records of 16 patients (22 days -10 years of age) with reversal sign were reviewed. Sonography was done in 7 patients. We divided our patients into three groups; group I, acute reversal: reversal sign on initial CT scan at presentation; group II, intermediate group: diffuse edema initially and reversal sign on 2-22 day follow-up CT scan; group III, chronic reversal: diffuse brain atrophy and/or cystic encephalomalacia with reversal sign. There were 8 trauma, 6 hypoxia/anoxia and two others. All acute and intermediate group patients had respiratory problems requiring ventilator support and intensive care. In three of four patients who died, autopsy findings were consistent with hypoxic/ischemic encephalopathy. Surviving patients had profound neurological deficit. Sonographically, we have noticed hypoechogenicity of the brain parenchyma with relative hyperechogenicity in the region of the thalami. In conclusion, the reversal sign is a unique CT finding probably representing a pattern of diffuse hypoxic/ 
ischemic cerebral injury. It carries a poor prognosis with irreversible brain damage.

Cerebral interstitial adenosine and blood flow during bicuculline-induced seizures in piglets. T. S. Park, D. G. Van Wylen, R. Rubio, and R. M. Berne (Charlottesville, Va.)

While adenosine has been suggested as a metabolic mediator linked to regulation of cerebral blood flow (CBF), its role in regulating $\mathrm{CBF}$ in the neonate remains unclear. To determine if adenosine participates in regulation of $\mathrm{CBF}$ during seizures in the neonate, we studied changes of interstitial fluid adenosine concentrations (ISF ADO) and the effects of $\mathrm{O}_{2}$ supply on ISF ADO by the brain dialysis technique in the frontal cortex of newborn piglets subjected to bicuculline-induced seizures. The $\mathrm{O}_{2}$ supply was changed globally by changing mean arterial blood pressure (MABP) and locally by varying $\mathrm{PO}_{2}$ in artificial CSF perfusing the dialysis cannula. Sagittal sinus blood flow (CBF), cerebrovascular resistance (CVR), and cerebral oxygen consumption $\left(\mathrm{CMRO}_{2}\right)$ were also examined in the same animal. Seizures increased ISF ADO 7.9 fold $(p<0.05)$ when ictal MABP was maintained at preictal level and perfusate $\mathrm{PO}_{2}$ was $24 \mathrm{mmHg}$ (group 1, N=6). ISF ADO increased 11.8 fold $(p<0.05)$ during seizures associated with moderate systemic hypotension and the low perfusate $\mathrm{PO}_{2}$ (group $2 \mathrm{~N}=6$ ). By contrast seizures increased ISF ADO only 3-fold $(p<0.05)$ when perfusate $\mathrm{pO}_{2}$ was increased to $-182 \mathrm{mmHg}$ and ictal MABP was maintained at preictal level (group 3, N=8). When ictal MABP was elevated from the preictal level and the perfusate was rich in oxygen, seizures failed to increase ISF ADO (group 4, $N=7$ ). In groups I and 3 , the increase in ISF ADO during seizures was associated with significant increases in $\mathrm{CBF}$ and $\mathrm{CMRO}_{2}$ as well as significant decreases in CVR. These data suggest that in piglets the increase in $\mathrm{O}_{2}$ supply during seizures did not match completely the increase in $\mathrm{O}_{2}$ demand resulting in enhanced release of adenosine into the interstitial space.

Spinal sensory evoked potentials in the tethered cord. S. Yamada, T. Fuse, and W. Hayward (Loma Linda, Calif.)

There have been few reports on somatosensory evoked potentials in the tethered cord, which were obtained from scalp recordings. We recorded somatosensory evoked potentials from the lumbosacral cord in response to the stimulation of the peroneal or tibial nerve. The recordings were done before and after untethering simultaneously to the recording of redox study of cytochrome $a_{2} a_{2}$. These include ten patients of tethered spinal cord associated with thick filum terminale, lipoma or lipomeningomyelocele. Before untethering cytochrome $a_{2}$ was reduced and interneuron potentials were diminished in amplitude. After untethering procedure redox shifted toward oxidation and amplitude of interneuron potentials increased. These changes correspond to those noted in experimental teth- ered cord. We conclude that spinal cord evoked potential studies are useful for evaluation of the surgical effect of untethering.

Split calvarial bone grafting in the dog model. J. P. Laurent, I. R. Abbott, and W. R. Cheek (Houston, Tex.)

The use of split calvarial bone grafts was first described in the late nineteenth century, and they are being used with increasing frequency for repair of cranial defects and craniofacial anomalies. To data there has been no reported study on the strength of this graft nor on its long term viability. In this study, nine mongrel dogs were used, each dog receiving four $1.5 \mathrm{~cm}$ diameter trephine craniotomies. The bone plugs harvested were split through the diploe, returning a partial thickness plug to each craniotomy site. The remaining partial thickness plugs were tested, defining their resistance to fracturing using a Matco servohydraulic materials testing machine. A computer monitored the resistance imparted by the bone plug. At 90-150 days, Te labeled phosphate was injected intravenously, the dogs sacrificed and a bone scan performed. The plugs were harvested, absolute radioactive counts obtained, and the plugs were submitted for histologic study or strength testing. Bone scanning revealed increased activity within the plugs, on the average absolute counts showed activity twice that of samples from adjacent calvarium and in no case was the activity less. Histologic study showed osteoblastic activity in all plugs. The strength testing showed an expected drop by $50 \%$ when full thickness calvarium was split with no return toward the strength of the full thickness calvarium occurring over time. In conclusion, while one can expect a viable graft when using this technique, one must be cognizant of a persisting diminution in strength at both donor and recipient sites.

Clinical experience using the projection geometry technique and mean theory reduction for specific problem areas in craniofacial surgery. J. M. Nadell and P. M. Hendel (New Orleans, La.)

Since we first began using the projection geometry technique for the reshaping of large cranial plates in 1982, we have found that it has specific applications in several classic craniofacial syndromes. Specifically, the list includes Apert's syndrome, Crouzon's syndrome, Treacher Collins syndrome, and syndromes involving cranial synostosis. To date our experience has been in 58 patients. Our experience has demonstrated that if used properly this technique has many advantages. In particular it offers the surgeon absolute control of the shape of the skull giving a superior cosmetic and functional result. There is also both a major saving of operative time and a great reduction in the neurosurgical risk. This paper will demonstrate the specific craniofacial maneuvers in the correction of each of these syndromes that are best accomplished by this method. Special attention will be given to demonstrating the differ- 
ence between the two projection geometry patterns and the types of syndrome for which each is best suited. Specific attention will also be given to describe pitfalls we have encountered and steps to avoid them. Longterm follow-up results will be presented.

Microsurgical anatomy of the IV ventricle in children. J. M. Velasco-Siles, L. Delgado-Reyes, and F. Lopez-Lopez (Anahuac)

The great variety and frequency of pathology located in the infratentorial compartment in children, and especially in the IV ventricle, makes necessary a real knowledge of these structures. It is then possible to interpret correctly the radiological studies and to perfect the microsurgical procedures for this region. The present work studied 10 postmortem cases. Arteries and veins of the brain were filled with acrylic, and the ventricular system and cisterns infused with gelatin and contrast medium. A radiological analysis of the IV ventricle and the infratentorial cisterns was made with a very detailed description of the microsurgical anatomy in this area, and its relations with special emphasis on vascular morphology.

Increased hypoxanthine concentrations in CSF of infants with hydrocephalus. H. E. James, R. Bejar, O. A. Saugstad, and L. Gluck (San Diego, Calif.)

Hypoxanthine, the end product of purine metabolism, is very elevated in body fluids during hypoxia. Hypoxanthine was measured in the CSF of hydrocephalic preterm infants, to determine in what degree hydrocephalus is associated with anaerobic metabolism of the brain. The concentration ranged from 7.5 to $28 \mu \mathrm{mol} /(\mathrm{X}=14.3)$. With lumbar punctures or with VP shunts, the hypoxanthine fell to $2.0 \mu \mathrm{mol} /$ $(p 0.05)$. These findings indicate that ventriculomegaly creates a singificant degree of brain hypoxia in these patients. Untreated, it may interfere with cerebral metabolism and brain development. It is recommended that in the presence of progressive ventriculomegaly other than from atrophy, prompt treatment of the hydrocephalus be carried out to minimize the secondary effects of the ventricular enlargement.

Quantification of cerebrospinal fluid shunt flow rates: assessment of newly designed programmable pressure valve. M. Matsumae, O. Sato, K. Ito, and R. Tsugane (Isehara)

Shunt placement diverting CSF is a reasonably effective treatment for hydrocephalus, and is widely used. However, the procedure often requires shunt revisions because of its overdrainage or inadequately small CSF flow rate through the device, besides obstruction of the tubing. With regard to valve pressure, selection of an appropriate valve for a certain patient before surgery is not always a simple task. A competent valve selected at the time of implantation might no longer be warranted for the multiple physiopathological conditions of the patient as time passes. It is thus desirable to develop one single valve in which its pressure can be modified without revision of the valve system. The newly designed SOPHYSA valve comes in one model which can be programmed for one of three different pressure settings. This valve lies in the variation of the force exerted by a semi-circular spring in different positions of its curve. By rotating the attachment point of the spring, one is able to alter its resistance with respect to a fixed contact point. The valve was designed for the three usual pressure gradients: low, medium, and high pressures. The pressure changes can be achieved externally by means of a special magnet enabling precise adjustment of the pressure valve through the scalp.

Clinical application of a quantative shunt flow rate monitoring device. F. J. de Moura Theophilo and W. F. Schoener (Rio de Janeiro)

To determine easily drainage flow rates in a shunt treated hydrocephalic child, a monitoring system was assembled to detect post shunting CSF drainage quantitatively. After positioning 4 miniature $\mathrm{CdTe}$ radiation detectors (2-3 $\mathrm{mm} \varnothing)$ along the shunt pathway (extracorporeal), a microbolus $(0.005 \mathrm{ml}) \mathrm{Tc} 99 \mathrm{M}$ Pertechnetate $(50 \mathrm{uCi})$ was injected into the shunt reservoir or valve. Continuous recordings of count rates and integrating over preselected time intervals (max. 64 integrations per detector) were stored in a portable data storage. In close correlation to the flow rate time-activity-histograms indicated bolus transport-velocity through the subcutaneous shunt tubing. Accuracy of this method was proven by 20 phantom tests and turned out to vary within a range of less than $5 \%$ to the real flow rates. Under clinical conditions flow rates in 18 patients were calculated and follow-up tests in 33 cases were performed. Flow rates were found within an unexpected wide range and a decrease of shunt flow rates in the first five days post shunting was found. The above presented method has proven to be useful in detecting malfunction as well as confirming proper function of an implanted CSFshunt.

Morphologic and biochemical effects of ventriculo-peritoneal shunts in neonatal kittens. J. P. McAllister II, T. J. Lovely, D. W. Miller, and R. C. Truex, Jr. (Philadelphia, Pa.)

The neurologic deficits that persist in one third of all hydrocephalic children receiving ventriculo-peritoneal (VP) shunts indicate that neurons are irreversibly damaged. To determine the histologic and biochemical effects of VP shunts, hydrocephalus was induced in 4-10 day old kittens by intracisternal injections of $25 \%$ kaolin. Control animals received similar injections of saline. One group of hydrocephalic kittens was sacrificed 18-25 days post-kaolin, when the animals were near death. Another group, confirmed to be hydrocephalic by ultrasonography, received VP shunts 11-12 days after kaolin injection. These animals 
were allowed to survive for $30-45$ days post-shunt and were compared to both age-matched control and hydrocephalic animals. Post-shunt ultrasonography showed that the lateral ventricles had receded markedly, which correlated well with the generalized improvement of neurological signs. Grossly, kaolin filled the basal cisterns to the same extent in both shunted and hydrocephalic animals. However, the pattern of sulci and gyri, the thickness of the cortex and the size of the ventricles all returned to normal in shunted animals. The proximal portion of the shunt was located in the lateral ventricle. Nissl staining revealed a dramatic improvement in the cytoarchitecture of cortical areas 22 and 17/18. Most lamina were clearly visible and contained normal neurons. However, some neurons in layers V and III of area 17/18 were dark and pyknotic. These dark neurons may represent projection neurons that were subjected to axonal damage prior to shunting. Layer VI, which undergoes extensive neuronal degeneration in untreated hydrocephalus, appeared normal in the shunted animals. HPLC analysis indicated that VP shunts were only partially effective in restoring norepinephrine levels in the cortex. These results suggest that VP shunts only partially reverse the neuronal degeneration and biochemical alterations caused by hydrocephalus.

Management of hydrocephalus in children using a two step procedure for individual shunt selection. W. F. Schoener and F. J. de Moura Theophilo (Münster)

Regarding the fact that physiological and pathophysiological conditions of the intracranial content in hydrocephalic children can be described by means of mathematics or physics, a two step procedure to clinical management of hydrocephalus was developed and tested, giving respect to special infant bound circumstances. (1) Description and calculation of cerebral spinal fluid (CSF) hydrodynamic parameters - including reabsorption resistance, $R$; CSFproduction rate, $F$; and compliance at base line pressure $\left(\mathrm{P}_{0}\right), C$ - on the basis of a modified pressure volume test (PVT) were performed by positive or negative intraventricular volume shifts. PVTs during shunt surgery avoided extracorporeal drainage for several days and allowed reliability improvement of parameter calculation even after introduction of double lumen catheters or catheter tip transducers for intraventricular pressure monitoring. (2) Calculation of a theoretical compliance correcting baseline pressure $P$ and calculation of an outflow deficit at $d_{c}$ at $P_{c}$ gave way for the determination of hydraulic parameters to select a proper shunting device. Results from 31 patients who underwent CSF shunt surgery are presented and a possible role of this two-step procedure in the clinical management of hydrocephalus in children are discussed.

Use of the subcutaneous reservoir in the treatment of hydrocephalus in the pre-term infant. A. E. Marlin, S. Rivera, and S. Gaskill (San Antonio, Tex.)

The use of the subcutaneous ventricular reservoir in the treatment of post-hemorrhagic hydrocephalus was studied in a series of 50 patients. All of the patients were considered to be medically labile, and had failed conservative therapy consisting of lumbar punctures with or without Lasix or Diamox. Management of the hydrocephalus consisted of reservoir taps that varied in intervals and amounts depending upon the degree of ventricular dilatation determined by sonography and the signs of increased intracranial pressure. The majority of reservoirs were left in place for 1-2 months. The total reservoir infection rate was $4 \%$; both of these infections were felt to be preventable, and there have been no infections in the last 38 patients. After the patients were medically stable the reservoir was removed and a shunt was placed. Eleven patients died before shunt placement and seven patients died after shunting, representing a $36 \%$ mortality rate. In no case was a death related to the shunt, but rather reflected the medical lability of the patient population. Five patients (16\% of surviving patients) did not require shunting. The total shunt infection rate was $9 \%$. These results support the use of the reservoir as an effective means of protecting the cortical mantle and decreasing morbidity related to future shunt placement.

Alternatives to treat neonatal hemorrhage and hydrocephalus. R. W. Oberbauer, W. D. Müller, and H. Eyb (Graz)

Intracranial hemorrhage is a common disorder in preterm infants; its management and prognosis are gaining interest due to increasing survival rates. In the past four years at this university, neonatal hemorrhage was found in 248 individuals (birthweights: $1,000 \mathrm{~g}-2,500 \mathrm{~g}$ ), $82.5 \%$ of this cohort suffered their first hemorrhage within the first three days. The rate of mortality amounted $35.5 \% ; 32.5 \%$ of the survivors developed progressive hydrocephalus - some $18.9 \%$, eventually turned out to require CSF diversion procedures, 5 of which died in the follow-up. Rather poor results of developmental outcome in the follow-up resulted in different attempts of management: (1) endoscopic clot evacuation soon after hemorrhage; (2) acetazolamide treatment with or without CSF removal; (3) external ventricular drainage; or, (4) permanent CSF diversion. Prior to recent years, it has been our attitude to act surgically as soon as progressive hydrocephalus was evident. Results of this early treatment were all but encouraging due to the frequent necessity of revisions and a number of tough infections. With expectant treatment $(100 \mathrm{mg}$ acetazolamide $/ \mathrm{kg} / \mathrm{d}$, occasional CSF removals, careful observation of intracranial pressure and sonographic patterns), we feel much more comfortable, as the percentage of operated individuals and the total number of operative procedures/patient have been reduced. As to psychomotor development, the comparison of early and expectant treatment of progressive hydrocephalus did not reveal a statistically significant difference; however, one has to consider the number of concomittant factors possibly influencing later outcome. 
Outcome of shunted non-tumor hydrocephalus: a review of 576 patients. C. Sainte-Rose, D. Renier, A. Pierre-Khan, E. Hirsch, and J. F. Hirsch (Paris)

Five hundred and seventy six children presenting to the Hospital des Enfants-Malades, Paris, between 1975 and 1984, have been selected for review, as all their surgical operations were performed in the Service using surgical isolation. The overall mortality was $10.0 \%$, and $41 \%$ of the deaths were related to shunt infections. In the survival group the mean intelligence quotient (I.Q.), or developmental quotient (D.Q.), was 72. Fifty-one percent of these patients had an I.Q. above 80. Sixty-two of the children had neurological deficits; $26 \%$ had epilepsy. The aetiology of the condition, the family environment and the occurrence of epilepsy were found to be related to the outcome. The first assessment of development quotient significantly relates to later evaluation of intelligence quotient. Ventricular dilatation, shunt infection and shunt malfunction did not show a direct correlation to I.Q. in this series.

Intracranial arachnoidal cysts in children. M. Ramos-Peek and F. Rueda-Franco (Mexico City)

Fourteen cases of intracranial arachnoidal cysts are presented; 9 males, 5 females, ages between one day and 11 years, with a median of 2.1 years. Symptoms were mainly restlessness in 8 patients, vomiting in 4 , seizures in 3 , headache and mild mental retardation in 2 . Signs were macrocrania in 10, enlarged anterior fontanel in 10, papilledema in 1. Associated findings: 3 patients, meningomyelocele; 2 , dysmorphic syndrome; 2 , neurofibromatosis and both presented complaining of deformity in the frontal bone and pulsatile exophthalmos; 1, lumbar aplasia cutex; and 1 lumbar ectopic stomach and pancreatic tissue. Skull X-rays showed 9 craniofacial disproportion, 2 frontotemporal bulging, 2 were normal, and 1 showed signs of intracranial hypertension. Cerebral ultrasound was performed on $7 \mathrm{pa}$ tients and all of them showed hydrocephalus and a cyst. Ventriculography was performed on 7 patients, 4 confirmed the cyst and 3 had hydrocephalus. Computed tomography gave the correct diagnosis in all 14 cases. The locations of the cysts were supratentorial: 6 over the quadrigeminal plate; 2 frontotemporal; 1 right frontal; 1 right parietal; and 1 parieto-occipital. Some were infratentorial: 2 in the midline and 1 in the left cerebellopontine angle. Surgical treatment for the cyst was: drainage to peritoneal cavity in 7 cases; resection of cyst in 4 cases; communication to lateral ventricle in 2 ; and communication to the cisterna magna in 1 . Ten patients required ventriculo-peritoneal shunt. The pathological study confirmed arachnoid membrane in 8 cases. The complications were CNS infection in 6 patients, and 3 others needed shunt revision. Follow-up has been from 30 days to 10 years with a median of 1.8 years; 2 patients have been lost during follow-up. Three developed normally, 4 have mild retardation, 3 moderate retardation, 3 have profound deficit with spasticity, and 1 died from aspiration on the fifth day after operation.
Hemispheric arachnoid cysts. C. di Rocco, M. Caldarelli, and A. Ceddia (Rome)

Out of 44 supratentorial arachnoid cysts in children surgically treated at the Neurosurgical Department of the Catholic University Medical School, Rome, in the period 1978/ 1986, four occupied the entire hemicranium. These hemispheric arachnoid cysts showed some particular characteristics besides the enormous extension, such as the asymmetrical enlargement of the ipsilateral hemicranium, and the surprising lack of clinical manifestations, in spite of the impressive distortion and contralateral dislocation of the entire brain. In spite of the removal of the lining of the cyst and/or the drainage of the cystic cavity, the re-expansion of the brain occurred very slowly in all the cases, reaching an almost complete normalization only in three cases. However, psychomotor development has been normal in all the subjects.

Cysts of the central nervous system. M. Mirko, R. Petar, B. Roza, and M. Danica (Skopje)

In the last 20 years (1967-1986), we have operatively treated 93 children with intracranial cystic lesions, from which 54 cases were congenital arachnoidal cystic lesions, 25 parasitic cystic lesions (echinococcus) and 14 arachnoid post-traumatic cystic lesions. Arachnoid cysts are intraarachnoid collections of cerebrospinal fluid, congenital in origin. They account for about $1 \%$ of all atraumatic intracranial cystic lesions. The localization of the arachnoid cysts in our series were in subarachnoid space, temporoSylvian region, ventricular system, and the posterior fossa. Neurological manifestations were worsening hemiparesis, palsy, epileptic fits, retardation, and change in consciousness complicated with hydrocephalus. Diagnosis was established on the basis of the physical examination of the children and paraclinical investigations. At first, we used plain X-rays of the skull (54 children - we found macrocephaly with enlargement and thinning of the vault in 21 cases, bulging in 16 cases, and CT scan (1978), gamma cisternography and arteriography. The surgical treatment consisted of craniotomy, opening and excision of the cystic membrane, either followed or not with cysto-peritoneal shunt. The follow-up ( 1 to 15 years) determined our satisfaction with the surgical treatment, since in the control period the clinical manifestations, such as stable child development, and brain position in its normal place in the endocranium improved.

The differentiation of posterior fossa cystic malformations: correlation of imaging with anatomical observations. D. $M$. Klein, G. Bennett, and F. G. Seidel (Buffalo, N.Y.)

Dandy-Walker malformation, posterior fossa "arachnoid" cyst, and enlarged cisterna magna can share common radiographic characteristics, and their differential diagnosis remains difficult despite modern imaging techniques. 
Dandy-Walker malformation can be found in various gradations of severity and is best defined as a dilatation of the furth ventricle associated with vermian dysgenesis. Reports of "arachnoid" cyst of the posterior fossa encompass a variety of histology, including glio-ependymal forms implying neural origin. In eight cases, cystic malformations of the posterior fossa were evaluated radiographically, including computed tomography (CT), and the efficacy of these studies in differential diagnosis was compared to that of magnetic resonance imaging (MRI), with particular attention to sagittal midline sections. In four of these cases, diagnostic studies were correlated with operative and microscopic progression of transitional forms between typical DandyWalker malformation and "arachnoid" cyst, linking these entities and supporting the developmental rather than obstructive origin of the Dandy-Walker malformation. We conclude that: (1) gross and microscopic observation of transitional forms suggests that posterior fossa glial-ependymal cysts and Dandy-Walker cyst may be part of a single continuum of cerebellar malformations; (2) unless cerebellar hypoplasia is extreme, differentiation of Dandy-Walker from "arachnoid" cyst and enlarged cisterna magna can be uncertain by $\mathrm{CT}$, with or without Metrizamide injection; (3) MRI, particularly in midsagittal cuts, is the best single differential study for these entities, but even MRI may fail to identify minor transitional forms with certainty.

Posterior fossa arachnoid cyst. Clinical, neuroradiological and surgical features. K. Sato, H. Arai, K. Yaguchi, and S. Ishii (Tokyo)

We have observed 14 cases of retrocerebellar cyst, 3 cases of unilateral cerebellar hemispheric cyst, and 2 cases of cerebellopontine angle cyst. These cases were subjected to various neuroradiologic tests, including sagittal reconstruction CT, RI- and/or CT-cisternography, angiography and others, when necessary. Seventeen out of these were treated surgically by an excision of the outer wall of the cyst, and followed by cystoperitoneal shunt in 15 of the 17. Taking account of radiological and histological features of the cysts, the 19 cases of the posterior fossa cyst were retrospectively classified into communicating or non-communicating intra-arachnoid cyst (7), Blake's pouch cyst (2), Dandy-Walker cyst (1), Dandy-Walker variant (2), glioependymal cyst (1), enlarged cisterna magna with communicating hydrocephalus (1), IV ventricular diverticulum (1), and large cisterna magna (4). In 10 out of the 17 surgically treated cases, postoperative disappearance of the cyst concommitant with re-expansion of the cerebellum and improvement of clinical problems were noted. From these retrospective analyses of the cases with posterior fossa cyst, the following conclusions were obtained: (1) occurrence of posterior fossa intra-arachnoid cyst, previously considered a rare pathological condition, was found to be more frequently encountered than had been thought and was found to be a surgically treatable disease; (2) though cases with IV ventricular cyst have been categorized into Dandy-
Walker cyst, Dandy-Walker variant, and Blake's pouch cyst, in the present study distinct neuroradiological differentiation into 3 of these categories remains uncertain; (3) the surgical results seemed not always directly correlated to the aforementioned cyst-classification; (4) the following neuroradiological findings including (a) either occipital bossing or petrosal scalloping with distortion or obliteration of CSF cisterns of the posterior fossa, (b) compression and deformity of the brain surrounding the cyst, (c) RIand/or CT-cisternographic findings suggestive of disturbance in intracystic CSF circulation, and (d) non-communicating cyst, seemed to indicate surgical treatment of the posterior fossa cyst.

Cavernous malformations (angiomas) in children. D. Rigamonti, H. Rekate, H. Pittman, and R. F. Spetzler (Phoenix, New Mex.)

Cavernous malformation of the brain, one of four types of cerebral vascular malformations, have been described as relatively benign lesions (clinically) and are most commonly diagnosed in the third and fourth decades. Our series includes 25 patients with histologically verified cavernous malformations. Thirteen presented during their first and second decade. Cavernous malformations appeared on magnetic resonance (MR) images as areas of mixed signal intensity, with a surrounding rim of decreased signal intensity on $T_{2}$ weighted images. When small, they appear as areas of decreased signal intensity - "black dots" on $T_{2}$ weighted images. Familial occurrence, previously thought to be exceptional, was encountered in $50 \%$ of the cases when MR scans of a patient's immediate family members were obtained. Of the 13 children operated upon for these lesions, $46 \%$ presented with seizures, $46 \%$ with neurological deficits, and $8 \%$ with signs of increased intracranial pressure alone. Our review indicates that cavernous malformations are most frequent in the pediatric population and are not necessarily benign lesions. Cavernous malformations are a nosological entity characterized by two forms; sporadic and familial. The familial form is transmitted in an autososmal dominant fashion. MR is the imaging modality of choice for the diagnosis of cavernous malformations and for screening suspected family members.

Cerebral cavernous angiomas in children. C. Mazza, R. Scienza, M. C. Zeppetti (Verona)

Cavernous angiomas are considered to be a rare pathology and, moreover, to occurr prevalently in adults. On the contrary, up to date methods of neuroradiologic research such as CT and, above all, MRI have shown greater frequency of such lesions in the pediatric age as well. In our Department, we observed 13 patients suffering cavernous angiomas whose age ranged from 7 to 16 years old, 12 diagnosed after 1982. Two patients were brothers. The most frequent symptom was hemorrhage (11 cases), while epilepsy was the only symptom in two cases. Lastly, two cases 
showed epileptic attacks at the beginning, and afterwards suffered hemorrhage. The frontal region turned out to be the more frequent site followed by the parietal. One of the patients presented with many supratentorial angiomas and one of them caused an hematoma. All of our patients underwent cerebral angiography, which in no case showed pathologic blood vessel circulation as sometimes described in scientific reports. In more recent cases, cerebral angiography carried out with DSA at a very slow pace, gave a negative result too. CT carried out in 12 cases resulted quite positive. In particular, patients with hemorrhage outlined a pattern which is characteristic: contrasting image of the hematoma and inside of it a hyperdense nodule due to the angioma which is plain after contrast infusion. The MRI carried out in 8 cases was always diagnostic, giving many peculiar images. Eleven patients underwent an operation for the extirpation of the whole cavernous angioma. The follow-up is considered excellent for all of them; excepting one patient who still shows an hemiparesis. Two of our patients were not operated upon because the cavernous angiomas were inside the brainstem and the basal nuclei. The authors conclude: (1) such lesions are much more frequent than we had considered; (2) they give neuroradiologic images which are very characteristic with CT and MRI; (3) operation is quite easy, at least in neurosurgically accessible sites; (4) it is necessary to examine thoroughly and repeat, if required, all of the neuroradiologic exams in all of the patients who are hospitalized with an intracerebral hemorrhage.

The surgical management of moyamoya disease in childhood. H. J. Hoffman, R. Griebel, S. Chuang, and H. Schutz (Toronto, Ont.)

Moyamoya disease is a progressive disorder affecting the intracranial internal carotid arteries which occurs predominantly in childhood. The clinical course of twentythree children with confirmed Moyamoya disease seen at The Hospital for Sick Children, Toronto, during the years 1971-1985, have been reviewed. Only three of these children were of oriental origin, eighteen were Caucasian. Three patients had neurofibromatosis, one had Down's syndrome and one patient had received radiation for an optic nerve glioma. Three of the patients were siblings of German Mennonite parentage. Fifteen of the twenty-three patients underwent one or more revascularization procedures. Six cerebral hemispheres were revascularized in five patients with the superficial temporal artery to middle cerebral artery (STA-MCA) bypass. Thirteen patients underwent an encephalo-duro-arterio-synangiosis (EDAS). The twenty-three patients have been followed for periods of time ranging from six months to fifteen years (mean 3.5 years). Two of the untreated patients have died. Of six surviving untreated patients only one remains neurologically intact and she continues to have headaches and TIA's. Two patients were treated only with STA-MCA procedures and both of these patients have had excellent results. Three pa- tients and both EDAS and STA-MCA procedures and two of these patients had excellent results and one patient has stabilized. The remaining ten patients were treated only with an EDAS procedure. Our results in the children who have had revascularization procedure are impressive. Not only does revascularization appear to prevent strokes and transient ischemic episodes over the time period studied, but it reverses fixed deficits in a significant percentage of the cases. The STA-MCA anastomosis provides an immediate blood supply to the ischemic hemisphere, and may be the effective alternative in infants and young children whose vessels are small. Our early data suggest that opening of the arachnoid beneath the transposed vascular cuff enhances the formation of pedicle to cortical anastomatic collaterals.

Moyamoya disease: can MRI predict the prognosis. Y. Maki, T. Nose, T. Enomota, and Y. Tomono (Ibaraki)

The moyamoya disease is a progressive cerebrovascular obstructive disease of unknown etiology, characterized clinically by a variety of symptoms due to transient ischemia, infarction, intracerebral hemorrhage, subarachnoid hemorrhage, and global ischemia. The diagnosis itself is easy by angiography, but the vulnerability of the brain to the slightest additional ischemia is such that one wishes a less threatening technique. For this purpose, we studied the magnetic resonance imaging (MRI) of moyamoya disease ( 5 children and 8 adults), other cerebral diseases, and those with slightest symptoms. The most striking feature of the disease was periventricular high intensity (PVHI) in early stages of life, which usually appear after 40 years and in relatively younger ages in those with vascular diseases. Other than PVHI the MRI demonstrated atrophy, massive infarction and lacunar formation.

The variations of pediatric arteriovenous malformations. R. P. Humphreys, E. B. Hendrick, and H. J. Hoffman (Toronto, Ont.)

Hemorrhagic stroke in a child, at least in this review, is more likely to be due to bleeding from an arteriovenous malformation than a ruptured intracranial aneurysm. This process, which also distinguishes the child from the adult, is characterized by a number of other differentiations, apparent from this review of 105 children studied at The Hospital for Sick Children (HSC), Toronto. More children bleed from their malformations (77\%) and fewer suffer chronic seizures $(15 \%)$ than adults. In that regard, there are remarkable similarities between the HSC review and those reported by others. The HSC study concludes that the mortality from any hemorrhage is $24 \%$. As with intracranial aneurysms, AVMs in children can be scattered throughout the brain, often in awkward locations. There are an unusual number $(21 \%)$ located below the tentorium. Wherever possible, total excision of the malformation must be attempted, with the expectation that the child's brain vasculature may be more resilient to operative intrusion. Six- 
ty-four percent of children receiving operative care can be expected to be neurologically intact after surgery. The operative mortality of $8 \%$ is influenced by the severity of the subarachnoid/intraventricular hemorrhage, as all patients were clinical grades 4 or 5 .

Arteriovenous malformations in children. D. Fong and S.-T. Chan (Hong Kong)

Thirty-nine patients, all below the age of 16 , suffering from arteriovenous malformations (AVM) in the central nervous system were studied retrospectively. All had unilateral lesions except one. Of these 40 AVMs, 39 were intracranial and the other was spinal. Surgical extirpation was carried out, if possible, after assessment by angiography and computed tomography. Inoperable cases were managed conservatively. The fact that AVMs do grow was demonstrated. From the promising results of the operated cases, it is strongly believed that total surgical excision is the treatment of choice. Importance of microsurgical approach cannot be exaggerated.

Cerebral AVM in children. C.-L. Lapras, J. N. Guilburd, J. Guyotat, and J. D. Patet (Lyon)

Sixty cases of cerebral AVM were treated in children ranging from 10 days to 15 years. Eighty percent of the cases presented as intracranial hemorrhage, $10 \%$ had seizures and 2 cases had heart failure. On arrival, 23 cases were alert, 31 obtunded, 6 comatose. Fifty-nine patients underwent surgery: 50 total removal; 9 partial removal or clipping. Six patients died (10\%): 3 were related to surgery; 2 to late re-bleeding, and one to embolization performed after partial removal. One of 50 cases with total removal bled later on. Total removal was achieved after one surgery in 45 cases, after two in 4 cases, after three and a hemispherectomy in 1 case. Forty-five percent of patients treated by total removal have no symptoms, $40 \%$ have a mild neurological deficit with a normal life. Of 9 partial removal, one died during surgery, 4 bled again, 2 had a slow but sustained aggravation, one has a short follow-up, only one is in good condition after 11 years. Of 3 patients treated by embolization after partial surgery, one died, one is badly and the other mildly handicapped. Analysing the relatively bad results of partial treatment of AVM in children, we advocate trying a total removal, even for large and deep malformations and even if a second surgery is needed. Embolization has to be kept as the last alternative. The dangers of partial clipping versus conservative treatment are emphasized.

Arteriovenous malformation of the brain in children. Review of 49 cases. G. Lena, C. Raybaud, L. Genitori, E. Empime, and M. Choux (Marseille)

During the last 17 years, 49 arteriovenous malformations of the brain were observed in children under 15 . We ex- cluded from this study 10 aneurysms of vein of Galen and 2 dura mater AVM. Eight cases (16.3\%) concern infants, but more than $50 \%$ of the cases are between 10 and 15 years old. Female predominance is important: 31 cases (63.3\%). Thirty-one patients $(63.3 \%)$ presented with intracranial hemorrhage, 12 patients $(24.5 \%)$ were in comatose state. Only 5 patients $(10.3 \%)$ with seizures alone were observed. The AVM was supratentorial in 45 cases (91.8\%) and infratentorial in 4 cases $(8.2 \%)$. Total surgical excision or clipping of the AVM was performed in 44 patients $(89.7 \%)$ and radiotherapy in 1 patient; 4 patients $(8.3 \%)$ were not treated. The results are excellent or good in 42 patients $(85.5 \%)$, even in bad risk grade; 5 patients $(10.3 \%)$ present with sequelae and 2 patients $(4 \%)$ died.

\section{Posters}

Use of bean bag for craniosynostosis surgery in modified prone position. T. S. Park, M. M. Harris, S. L. Henson, and J. A. Jane (Charlottesville, Va.)

A modified prone position reported in 1985 allows for a calvarial access from the supraorbital ridge and the occipital bone adjacent to the foramen magnum [1]. While this position appears to gain increasing popularity among pediatric neurosurgeons, a proper frame for the position has not been available. We recently used the bean bag (VacPac, Olympic Medical, Seattle, Wash.) to achieve the modified prone position intraoperatively. The bean bag was found to support safely the face of infants who undergo craniosynostosis surgery in the modified prone position.

1. Park TS, Haworth CS, Jane JA (1985) Modified prone position for cranial remodeling procedures in children with craniofacial dysmorphism: technical note. Neurosurgery $16: 212-214$

Stereotactic radiosurgery for pontine AVM. Case report. M. Kurisaka, K. Mori, T. Arimitsu, and A. Moriki (Kochi)

Since the 1950s, total resection of all pathological arteriovenous shunts has been considered the only reliable surgical treatment for AVM's. But surgery may involve a high degree of risk if the location of the AVM is near the brain stem. Recently we had a case of pontine AVM, and experienced stereotactic radiosurgery in this case. The patient, a six year-old boy, developed a sudden loss of consciousness followed by right-sided earache and vomiting. CT scan revealed hematoma in the ventricular system and angiography showed an AVM in the pons. After suboccipital craniectomy and two ventricular drainages, the patient has recovered slowly but steadily. For steriotactic radiosurgery, we took him to Karolinska Institute in Stockholm. Target point was decided in the centrum of the $\mathrm{AVM}$, and its $\mathrm{X}, \mathrm{Y}, \mathrm{Z}$ coordinates computed. Using a collimator of $8 \mathrm{~mm}, 40 \mathrm{~Gy}$ were given to target. After nine months, follow-up angiography was performed and partial obliteration was proved. 
Endoscopic findings in pediatric hydromyelia? A degenerative phenomenon. J. R. Mawk and J. McConnell (Omaha, Neb.)

Endoscopy of hydromyelic cavities was performed using a $4.5 \mathrm{~mm}$ external diameter pediatric bronchoscope during the course of detetherings and hydromyelio-subarachnoid shunts. Findings within such cavities include: (1) membranes; (2) vertical, oblique and horizontal trabeculation of the wall of the cavity; (3) vessels upon trabeculations; (4) relative pallor of the more involved areas of the cord; (5) sessile, glioneuronal "polyps" or tissue remnants which waft in CSF currents; (6) a spectrum of nervous tissue destruction which does not correlate with size of cavity or cord level. Hydrostatic and hydrodynamic factors alone may not explain the observed pathology. The spinal cord in certain syndromes (e.g., Chiari II malformation) may be unusually sensitive to pressure, or may be undergoing intrinsic degeneration, so that hydromyelia is an ex vacuo phenomenon.

Intracranial arachnoid cysts with multiple anomalies. $\mathrm{H}$. Takahashi, L. N. Sutton, L. Schut, and S. Nakazawa (Philadelphia, Pa.)

Intracranial arachnoid cysts are benign developmental cavities which have provoked controversy among neurologists and neurosurgeons. Robinson asserted that middle cranial fossa cysts formed in response to temporal lobe agenesis in 1971. However, subsequent work has suggested that arachnoid cysts are primary congenital lesions within the arachnoid membrane. Recently, we have experienced two cases of intracranial arachnoid cysts with other multiple anomalies. These two cases are very suggestive for considering the pathogenesis of intracranial arachnoid cysts. Both patients were full-term males born by normal spontaneous vaginal deliveries. At birth, the babies were noted to have large heads, cleft lips, cleft palates and hypertelorism. In addition, one baby had an atresia ani. Computerized axial tomograms of the brain revealed huge right temporo-parietal arachnoid cysts in both patients. One of them had an arachmoid cyst which communicated with the ventricular system, but the other had a non-communicating arachnoid cyst. Right ventriculoperitoneal shunt (communicating cyst) and cyst-peritoneal shunt with craniotomy (non-communicating cyst) were performed for increased intracranial pressure. Agenesis of right temporal, occipital lobes, and tentorium cerebelli were recognized by craniotomy. These anomalies are correlated with abnormalities of following gestational ages: atresia ani, 4-5 weeks; cleft lip and palate, 5-7 weeks; agenesis of temporal occipital lobes and tentorium cerebelli, 6-7 weeks. On the other hand, choroid plexus appears secretory at gestational age 7 weeks. In conclusion, some intracranial arachnoid cysts probably arise during the earliest stages of embryogenesis (5-7 weeks).
Frequency index profile analysis of pulsed Doppler carotid artery flow in hydrocephalic and non-hydrocephalic infants. J. C. Anderson and J. R. Mawk (Omaha, Neb.)

We hypothesized that carotid artery blood flow would be diminished by hydrocephalus, and conversely that measurement of the blood flow would allow a relative assessment of severity of hydrocephalus. Pulsed-Doppler waveform analysis was carried out using a personal computer by tracing the observed waveform on a digitizer pad. Use of a program developed by one of us (JCA) allowed conversion of the waveform to a number, termed the frequency index profile (FIP). We studied 30 normal infants to form a control group. The FIP was also determined in 29 infants with hydrocephalus requiring shunting, 30 infants with ventriculomegaly not requiring a shunt and 62 infants with various forms of brain insult not associated with enlarged ventricles. We found that carotid artery blood flow by this technique is usually normal in hydrocephalics, irrespective of how advanced this condition may be. On the other hand, high FIP scores routinely identified anoxicischemic encephalopathy when other methods failed to do so. The technique may have some value in assessing conditions co-existent with hydrocephalus; poor outcome following shunting of certain categories of patients may also be predicted in this manner.

Outpatient monitoring of headache complaints in shunt patients. R. Abbott, J. H. Wisoff, and F. J. Epstein (New York, N.Y.)

A technique has been devised which allows us to monitor patients' intracranial pressure over several hours in an outpatient setting avoiding needless hospitalization and more invasive diagnostic or therapeutic maneuvers. A 23 gauge butterfly catheter is introduced percutaneously into the shunt dome and then CSF is aspirated to eliminate air in the line. A seal devised by Camino Labs is then placed on the end of the catheter and their fiber optic pressure catheter introduced through the seal into the fluid filled catheter. The pressure monitoring system is then attached to a chart recorder to allow longitudinal recording of pressures. The responsiveness of the system is assessed by monitoring ICP change during jugular compression and the ability of the system to record normal ICP pressure waves. The patient is instructed to mark on the chart recorder any symptoms to allow correlation of these symptoms with ICP. During the period of monitoring the patients are encouraged to change the elevation of their head, to sit or lie and to try to duplicate any maneuvers which they feel might result in the triggering of noxious symptoms. The technique of the system will be discussed as will several of the case histories of patients monitored with the system.

New instrument "skull punch" for craniotomy in neonates and early infants. N. Morota, K. Sakamoto, and N. Kobayashi (Kobe)

We have invented a new instrument, called "skull punch" in cooperation with Mizuho Ika Company, Tokyo, Japan. 
This instrument is useful and safe for making a burrhole for craniotomy in neonates and early infants. This instrument, made of stainless steel, is columnar in shape and $9 \mathrm{~cm}$ in length. Two sizes in the diameter of the tip are available: $6 \mathrm{~mm}$ and $7.5 \mathrm{~mm}$. With a back and forth rotatory motion of the punch, we can make a burr hole and gain a small round bone button. The skull punch has the following characteristics: (1) it is easy to control the power while making a burr hole; (2) we can make a burr hole safely enough not to injure the dura; (3) osteoplastic craniotomy is possible to apply small bone buttons at the site of burr holes; (4) sterilization can be done as with other regular instruments. Operative cases of neonates and infants in which this device was used will be presented.

Tethered cord - clinical presentation, urological findings, surgical management and outcome. $K$. Muraszko, P. W. Carmel, E. Reilly, and T. W. Hensle (New York, N.Y.)

With the development of improved radiologic techniques, particularly the MRI, an increasing number of children have been identified to have tethered cords. We review a series of 30 patients treated at the Neurological Institute in the last five years. Thirty two percent of the patients presented with meningomyelocele; $68 \%$ presented without associated meningomyelocele. The ages ranged from infants to 38 years of age. Urologic symptoms were noted by more than $80 \%$ of the patients reviewed. Two of the meningomyelocele group presented with preoperative enuresis and $55 \%$ of the non-meningomyelocele group presented with symptoms of incontinence or enuresis. Dysuria, frequency, and decrease in force of stream were also commonly noted. Neurological findings included sensory loss in the sacral dermatomes, gait abnormalities, and back pain.

Association of BK virus with pediatric brain tumors. S. Pezzotta, V. Silvani, P. Gaetani, D. Locatelli, and N. Bonfanti (Pavia)

BK virus (BKV) is a human papovavirus first isolated from the urine of an immunosuppressed renal transplant recipient. BKV infection has a worldwide distribution, since specific antibodies are found in nearly $80 \%$ of adults. Seroconversion, generally, occurs during childhood and primary infection, sometimes associated to pathological manifestations, is followed by a persistent, latent infection which is reactivated under conditions of impaired immunologic response. In vitro, BKV transforms both several animal and human cells. When it is inoculated either intracerebrally or intravenously in immunosuppressed hamsters, 73 to $88 \%$ of the animals develop ependymomas, choroid plexus papillomas, tumors of pancreatic islets and osteosarcomas. Using blot hybridization techniques we have studied BKV DNA and RNA in 3 cases of pediatric brain tumors. The tumor portions surgically removed were immediately frozen in liquid nitrogen and stored at $-80^{\circ} \mathrm{C}$ until used. In the case of ependymoma BKV DNA and RNA have been identified while in the case of "yolk sac tumor" viral RNA was extracted but not DNA. In the third case (a metastasis of neuroblastoma), neither viral DNA nor RNA were detected. The presence of BKV specific RNA in some tumors suggests that the viral genome is expressed, although some discrepancies were observed between the presence of BKV DNA and the expression of virus-specific RNA. While tumors positive for BKV DNA and negative for RNA are acceptable because viral DNA may not be expressed, at present there is no clear explanation for the tumors that apparently express BKV RNA without containing viral DNA.

Intracranial arteriovenous malformation in infancy. J. C. Suarez and J. C. Viano (Cordoba, Argentina)

Fifteen children and adolescents with intracranial arteriovenous malformation, whose ages ranged between 1 day and 15 years, are analysed. In this series, the arteriovenous malformations were clinically discovered through encephalic hemorrhage in 9 cases, epilepsy in 3, subarachnoid hemorrhage in 1 , retarded maturity with epilepsy in one, and in the remaining patient through cardiac insufficiency at birth. Of the 9 patients with encephalic hemorrhage, in two cases it was situated in the cerebellum and in the remaining 7 in the cerebrum. Of these, 2 were in the frontal lobe, 2 in the parietal lobe, 2 in the parietoccipital region and one in the optic thalamus. The patient with mental retardation, epilepsy, had a large arteriovenous malformation fed by both anterior cerebral arteries and the middle and left posterior cerebral arteries, with drainage in the superior longitudinal and in the Galen vein. The child with cardiac insufficiency at birth had a massive aneurysm of the vein of Galen. The diagnostic procedures employed in this example were: computerised tomography of the cerebrum in 14 cases, cerebral arteriography in 15, and EEG in 4 cases. The treatment was surgical, radiation, and anticonvulsive drug. This last was the only treatment in the case with a very large AV malformation. The surgical treatment consisted of the evacuation of the hematoma and the resection of the AV malformation in one or two times. Radiation treatment was practised in two patients on whom gamma rays were applied. In one, the AV malformation was in the left optic thalamus, and in the other in the speech area. Four patients died, 2 after operation, 1 through a lesion of the brain stem from hematoma of the cerebellum, not operated on, and the final case through intraventricular hemorrhage, four months after surgery (the necropsy in this case revealed remains of $\mathrm{AV}$ malformation). The two deaths for surgical reasons were through aneurysm in the Galen vein and a thrombotic AV malformation, deep temporal, which caused a large cerebral ischemia. The general mortality rate was $26.6 \%$ and surgical $13.3 \%$. 
Spontaneous intracranial hemorrhage in children. J. C. Suarez and J. C. Viano (Cordoba, Argentina)

We analysed a series of 20 children whose ages ranged between 6 months and 15 years, with the majority over 5 years old and with 12 males to 8 females. The initial symptoms were paleness in one case, convulsions with multiple spontaneous hematomas in 2 , vomiting in 7 , sensory alteration in 7 , and headaches in 16 . The convulsions were general in 2 cases and of half the body in 2 . The symptoms and signs on admission were: bilateral papilledema in 5 cases; hemiparesis in 7; alteration in consciousness in 7; and the meningitic syndrome in 10 cases. The changes in consciousness varied from coma in 4 cases to stupor in 2 and 1 case of drowsiness. The etiology was cerebral tumor in 1 case, blood dyscrasia in 2 cases, aneurysms in 3 cases, unknown in 5 cases, and from arteriovenous malformation in 9 . These last were found in cerebellum in 3 patients and in the cerebrum in the remainder. The diagnostic procedures employed were pneumoventriculography in 1 case, lumbar puncture in 6 , electroencephalograms in 7, carotid arteriography in 7, cerebral panarteriography in 11, computerised tomographies of the brain in 16, and X-ray of the skull in 20 cases. The treatment was surgical in 10 cases, medical in 8 , radiation in 2 cases. These last were, in one patient with arteriovenous malformation in the left optic thalamus and in the other with AV malformation in the Broca area. Four patients died. There was no surgical mortality. The deaths were through hemophilia type $A$ in 2 cases, massive aneurysm rupture of the anterior communicating artery in one, and in the other case, through the rupture of an enormous arteriovenous malformation in the cerebellum. Of the 16 surviving children, only 4 are suffering from further problems; these are generalised convulsions, controlled by medication in 2 cases and hemiparesis with hemianopsia in 2 cases. These last correspond to the children with porencephalic post-surgical cysts.

Use of the $\mathrm{CO}_{2}$ laser in posterior fossa tumors of childhood. W. L. Robinson and M. Randal (Baltimore, Md.)

The ability of the $\mathrm{CO}_{2}$ laser to vaporize tissue, permit delicate and precise dissections, and provide a modicum of coagulation makes it an important surgical tool in the treatment of posterior fossa tumors in childhood. Attempts at extirpation of these all too common lesions often leads to exploration of not only the cerebellar hemispheres, but also the cerebellar peduncles, the IV ventricle, and the brain stem. An analysis of 92 consecutive pediatric brain tumors yielded 60 cases treated with the $\mathrm{CO}_{2}$ laser. All patients had surgery with the laser mounted to the microscope. The tumor distribution is as follows: astrocytoma (group I/II) 15, (group III/IV) 9; medulloblastoma 22; ependymoma 8 ; ependymoblastoma 3 ; hemangioblastoma 3. Gross total resections were achieved in $97 \%(50 / 50)$ and near total resections in 3\% (2/60). Operative mortality was $0 \%(0 / 60)$ with an operative morbidity of $7 \%(4 / 50)$. Average follow-up is 4.5 years with a recurrence rate of $7 \%$ $(4 / 60)$. Posterior fossa surgery in children was performed, and performed well, prior to the advent of the laser. We feel, however, that the use of the $\mathrm{CO}_{2}$ laser allows for a delicacy of dissection, i.e., a "no touch technique", that allows an approach to vital and retraction, manipulation, and vibration. 